\title{
The Impact on Global Warming of the Substitution of Refrigerant Fluids in Vapour Compression Plants: An Experimental Study
}

\author{
C. Aprea, A. Greco and A. Maiorino \\ Additional information is available at the end of the chapter
}

http://dx.doi.org/10.5772/48349

\section{Introduction}

The development of vapour compression refrigerating units was strictly related to the characteristics of the working fluid from the beginning of their commercial diffusion.

Initially, natural substances were employed, such as ethyl ether, methyl ether, dimethyl ether, carbon dioxide, ammonia, sulphuric anhydride and methyl chloride. Potential users of the refrigerating equipment were somewhat diffident because of their toxicity and/or flammability. Most first generation refrigerant fluids were retired for safety reasons. Some, such as ammonia and hydrocarbons, survived or were later revived for limited applications in which their risks were manageable, such as industrial or small-charge systems.

Apparently, all safety problems were overcome with the appearance of the first, non toxic, non-flammable chloro-fluoro-hydrocarbon (CFC). Further chloro-fluoronated compounds followed, originating either from methane or from ethane by partial HCFC or total CFC substitution of the hydrogen atoms.

The first global environment problem with the second generation refrigerants was the depletion of stratospheric ozone. The problem arises from destruction of ozone molecules in the upper atmosphere, primarily by bromine and chlorine from anthropogenic chemicals. The chlorine and bromine react catalytically to destroy ozone molecules, thereby reducing the natural shield from incoming ultraviolet-B radiation. Molina and Rowland [1] identified CFCs and HCFCs as a source for chlorine in the stratosphere and the potential for more serious ozone depletion, with projected growth in use of these chemicals. The index used to indicate the relative ability of a refrigerant or other chemical to destroy stratospheric ozone is the Ozone Depletion Potential (ODP). 
ODP is defined for any given substance as the ratio between the ozone consumption per unit mass released in the atmosphere and that consumed by the CFC R11 [2].

Chlorinated and brominated refrigerants, along with similar solvents, foam blowing agents, aerosol propellants, fire suppressants, and other chemicals are being phased out under the Montreal Protocol, a landmark international treaty to protect the ozone layer [3].

Therefore the Montreal Protocol forced abandonment of ozone-depleting substances (ODSs) as refrigerants in current vapour-compression refrigeration systems. CFCs have been banned since 1996, with HCFCs for interim use. Indeed, their ODP, though lower than that of the CFC, is different from zero [2]. The transition from HCFCs also is underway. The Montreal Protocol sets limits for the HCFC consumption (or cap), defined as production plus imports less exports and specified destruction: in 1996 (freeze at calculated cap), 2004 (65\% of cap), 2010 (25\%), 2015 (10\%), and 2020 (0.5\%) with full consumption phase-out by 2030 in non-Article 5 countries [3,4]. Individual countries adopted different response approaches. Most western and central-European countries accelerated HCFC phase outs, while the majority of other developed countries set limits by phasing out propellant and blowing agent uses early, requiring phase-out of R-22 by 2010, and then banning all HCFC use in new equipment by 2020. The schedule for Article 5 countries begins with a freeze in 2013 (based on 2009-2010 production and consumption levels) with declining limits starting in 2015 (90\%), 2020 (65\%), 2025 (32.5\%), and 2030 (2.5\%) followed by phase-out in 2040. Again, continued future use and service, even after 2040, are allowed for existing equipment employing HCFC refrigerants until otherwise retired except as restricted by national regulations. Exports from Article 5 countries into non-Article 5 countries are effectively restricted to meet the more stringent non-Article 5 schedules. To avoid separate domestic and export products and to exploit newer technologies derived from joint ventures and licensing agreements, some products in Article 5 countries incorporate replacements earlier than required.

The HFCs are a new family of substances that are candidates for substitution of both CFCs and HCFCs. HFCs, in fact, are entirely harmless towards the ozone-layer, since they do not contain chlorine.

Now a further problem must be considered, the so-called greenhouse effect stemming from the capture of infrared radiation by some components of the atmosphere $[5,6]$. The average temperature at the surface of our planet results from an equilibrium between incoming solar energy and heat radiated back into space. Most of the latter is in the infrared range of emissions. Gases that absorb this infrared energy enhance the greenhouse effect of our atmosphere, leading to warming of the Earth. Human activities have increased substantially the concentration of greenhouse gases. As a result, a substantial warming of the earth surface and atmosphere occurred that might adversely affect the natural ecosystem. Over the last hundred years, the mean temperatures have increased by $0.3-0.6^{\circ} \mathrm{C}$. Doubling the amount of carbon dioxide in the atmosphere is likely to yield a further temperature increase by $1.5-4.5^{\circ} \mathrm{C}[7,8]$. Refrigerants have been identified as greenhouse gases. The impact of a given greenhouse gas on global warming is quantified by its GWP (Global Warming 
Potential). GWP is defined as the mass of $\mathrm{CO}_{2}$ that would yield the same net impact on global warming as the release of a single unit $(\mathrm{kg})$ of the given atmospheric component. The GWP values used in this paper are relative to a 100 years Integration Time Horizon. Shorter integration periods emphasize near-term effects, while longer intervals better reflect the total impact of a release. Carbone dioxide is used as the reference chemical for GWPs because it is the one between natural gases with the greatest net impact. Other chemicals, including most refrigerants, are more potent as greenhouse gases.

In December 1997 more than 160 nations met in Kyoto, Japan, to negotiate binding limitations on greenhouse gases for the developed nations, pursuant to the objectives of the United Nations Framework Convention on Climate Change (UNFCCC). The outcome of the meeting was the Kyoto Protocol [9], in which the developed nations agree to limit their greenhouse gases emissions, relative to the levels emitted in 1990. The Protocol is subjected to ratification, acceptance, approval or accession by Parties to the Convention. Due to the voluntary characteristics of the Protocol some countries have no targets under the Protocol, but the protocol reaffirms the commitments of the Framework Convention by all Parties to formulate and implement climate change mitigation and adaptation programs. Even countries such as the United States of America that have not formally signed onto the Kyoto Accord have introduced their own plans to aggressively reduce greenhouse gas emissions.

National laws and regulations implementing the Kyoto Protocol differ from one another, but they typically prohibit avoidable releases of HFC refrigerants. In some countries, their use undergoes control and/or taxation. More recent measures (either already adopted or proposed) at local level (regional, national, municipal) are even more stringent. These restrictions are forcing the shift to a fourth generation of refrigerants with both ODP and GWP regulations [10].

In the field of the mobile refrigeration systems, the European Parliament already set a regulation of F-Gases phase out [11] that bans the use of refrigerants having GWPs exceeding 150 (based on 100 years integration time horizon). Such regulation begins in 2011, and will be effective for all air conditioners of new automobiles in 2017 .

However, the EU Parliament [12] rejected recommended measures that would have banned HFCs as aerosol propellants by 2006, as foam blowing agents by 2009 , and as refrigerants in stationery air conditioners and refrigeration by 2010 .

\section{The TEWI concept}

The US Energy Information Authority projects that world carbon dioxide emissions will increase from 25.028 million metric tons in 2003 to 33.663 million metric tons in 2015 and 43.676 million metric tons in 2030. Electricity actually causes more carbon dioxide emissions than all other anthropogenic sources. According to the Energy Industry Administration in the United States, electricity generates $39 \%$ of the total anthropogenic carbon dioxide emissions. These emissions are expected to grow by almost $45 \%$ over the next 25 years and grow to $42 \%$ of the total carbon dioxide emissions. 
In order to reduce the production of greenhouse gases, it is reasonable to assume that we should focus on the reduction of emissions in both electricity and transportation.

According to US Department of Energy [13-15], the largest single use for electricity is lighting (27\%) followed by cooling (refrigeration and air conditioning) at $15 \%$, as shown in Figure 1.

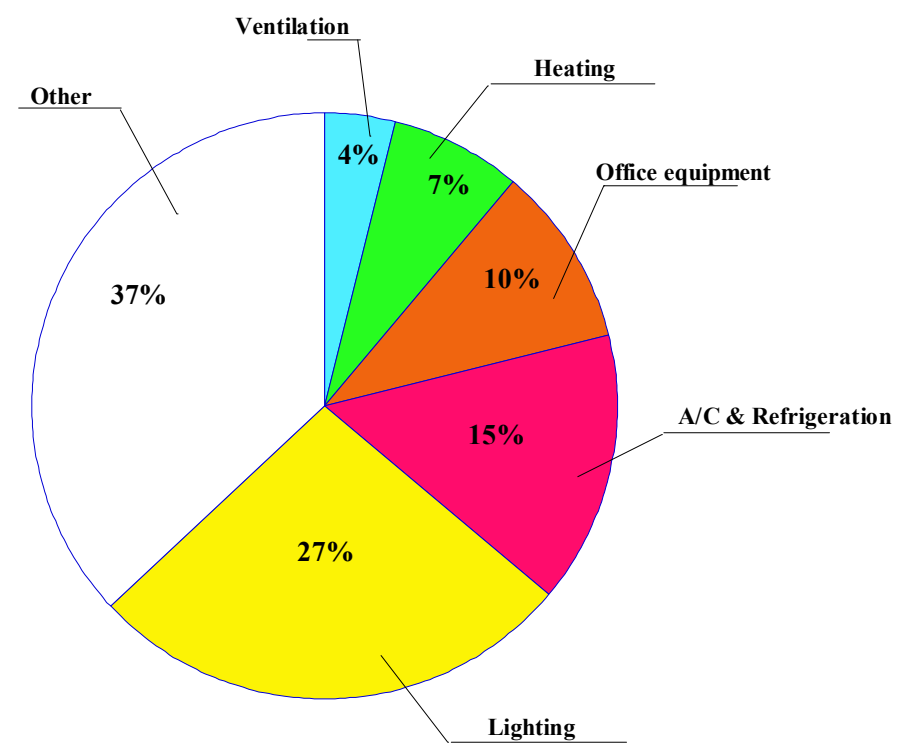

Figure 1. USA Electricity Consumption

Therefore in order to reduce the $\mathrm{CO}_{2}$ emissions caused by electricity a good option is to reduce the refrigeration and air conditioning emissions.

Vapour compression plants produce both a direct and an indirect contribution to global warming. The former depends on the GWP of refrigerant fluids and on the fraction of refrigerant charge which is released into the atmosphere during operation and maintenance, or is not recovered when the system is scrapped [16,17]. The indirect contribution is energyrelated. In fact, a vapour compression refrigerator requires electrical energy produced by a power plant that typically burns a fossil fuel releasing $\mathrm{CO}_{2}$ into the atmosphere.

The concept of total equivalent warming impact (TEWI) was developed to combine the effect of direct refrigerant emission with those due to energy consumption and the related combustion of fossil fuels for the electric energy production. TEWI provides a measure of the environmental impact of greenhouse gases originating from operation, service and endof-life disposal of the equipment. TEWI is the sum of the direct contribution of the 
greenhouse gases used to make or to operate the systems and the indirect contribution of carbon dioxide emissions resulting from the energy required to run the systems along their normal lifetime [18].

The TEWI is calculated as [19-21] :

$$
\begin{array}{lr}
\text { TEWI }=\mathrm{CO}_{2, \text { dir }}+\mathrm{CO}_{2, \text { indir }} & {\left[\mathrm{kg} \mathrm{CO}_{2}\right]} \\
\mathrm{CO}_{2, \text { dir }}=R C\left[P_{L}+\left(\frac{1-P_{R}}{V}\right)\right] V \cdot \mathrm{GWP} & {\left[\mathrm{kg} \mathrm{CO}_{2}\right]} \\
\mathrm{CO}_{2, \text { indir }}=\alpha \cdot \frac{\dot{Q}_{\text {ref }}}{C O P} \cdot H \cdot V & {\left[\mathrm{~kg} \mathrm{CO}_{2}\right]}
\end{array}
$$

The direct global warming effect of refrigerant fluids, stemming from the absorption they produce of long-wave radiations, depends on their GWP and on the fraction of refrigerant charge released into the atmosphere. The last is mainly due to leakage during the plant operational life time $\left(\mathrm{P}_{\mathrm{L}}\right)$ and to the residual amounts which, according to the current state of technology, are not recyclable and thus are released into the atmosphere when taking the plant out of operation (1-PR).

As already stated, the indirect contribution to TEWI consists in the so-called energy-related contribution. Indeed, an electrical refrigerator requires electrical energy from a $\mathrm{CO}_{2}$ releasing power plant that typically burns a fossil fuel. The amount of $\mathrm{CO}_{2}$ emitted is a function of the refrigerator COP, of the power plant efficiency and of the fuel used in the conversion plant that affect the emissions per unit energy converted [22]. When a fuel is burnt, energy is produced and carbon dioxide and other chemicals, mostly water, are produced. The ratio of $\mathrm{CO}_{2}$ emitted to the electricity generated differs according to the type of fuel used. Electricity is generated from a range of fuels including nuclear, gas, oil, coal and in some cases waste. Besides burning a fuel there are several other alternative to produce electricity like hydroelectric plants, wind power, geothermal energy sources, tidal power, photovoltaic panels etc.

The relationship between $\mathrm{CO}_{2}$ production and electricity generation may vary significantly, depending on the approach to be followed. In Figure 2 are reported the best values of $\mathrm{CO}_{2}$ emissions by primary energy sources taken from the reference providing the most recent data.

The typical power-plant technology adopted varies, therefore each country and each region inside each country has its own mix of primary sources for electricity generation. This mix can change significantly from country to country and even from one region to another in the same country.

The literature provides some indicative, average levels of $\mathrm{CO}_{2}$ release per $\mathrm{KWh}$ of electrical energy $(\alpha)$ for various countries [23-26]. Table 1 reports a values for different continents and, in each continent for different countries. In Table 1 are reported a range of $\alpha$ values and the "best value" for each country. 
Figure 3 illustrates the range of average country emission rates for several western European nations and compares those with relative percentages of electricity produced by each countries.

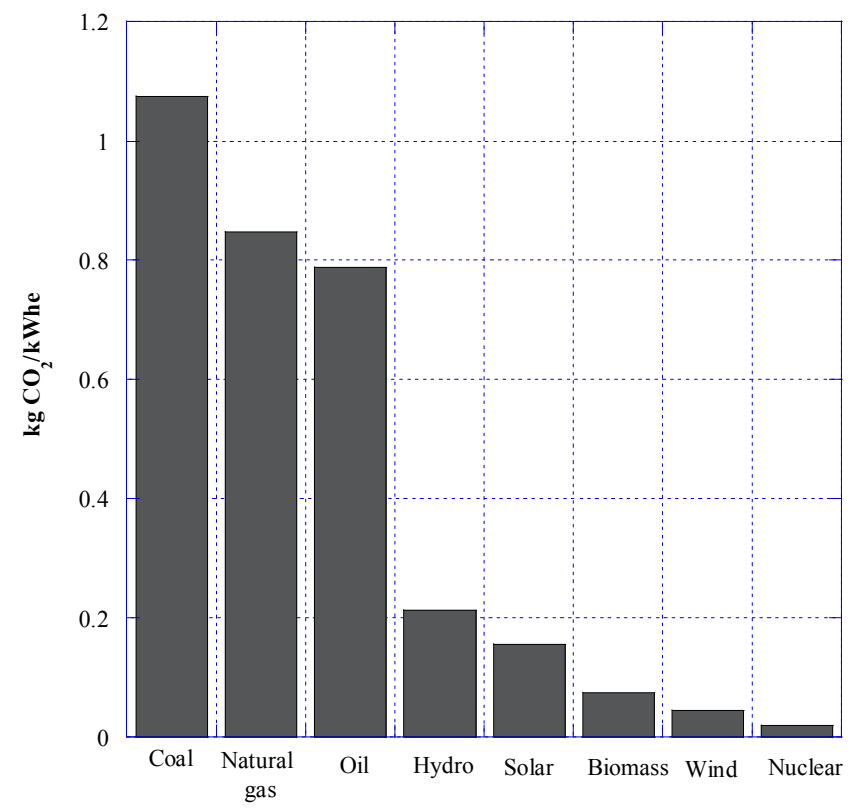

Figure 2. Average $\mathrm{CO}_{2}$ emissions by primary energy source.

In order to understand direct and indirect contributions impact on the greenhouse gases emissions typical household and light commercial refrigeration systems (cooling capacity below $1 \mathrm{~kW}$ at LBP ASHRAE conditions) were chosen for three representative market around the world (North America, Europe and Asia). Each system was tested following the energy consumption standards of each region and the results are presented in Table 2.

The concept of TEWI in this study is used to identify the global warming impact for each equipment and market region.

For calculation purposes an annual leakage rate of 5\% was adopted. This value was an intermediary value for household and light commercial applications. A mean value of life expectancy for refrigeration equipment in this study was 10 years. A recycling of $75 \%$ was considered for both household and light commercial applications. This means that $75 \%$ of the refrigerant charge is recovered at the end of the equipment useful life.

The results are shown in Figure 4.

In figure is also reported the influence of indirect and direct contribution to global warming in terms of $\mathrm{CO}_{2}$ emissions. The figure allows a comparison between the TEWI values for household and light commercial refrigeration systems. 
With reference to the previous study, in Figure 5 is reported the percentage of $\mathrm{CO}_{2}$ emissions of the direct and of the indirect contribution to global warming for each application.

\begin{tabular}{|c|c|c|c|c|c|c|c|c|c|}
\hline \multirow[t]{2}{*}{ Continent } & \multirow[t]{2}{*}{ Country } & \multicolumn{4}{|c|}{$\begin{array}{c}\text { Energy Mix by } \\
\text { Primary Source } \\
\%\end{array}$} & \multicolumn{4}{|c|}{\begin{tabular}{cc}
\multicolumn{2}{c}{ Total Energy } \\
Production $\quad \alpha\left(\mathrm{kgCO}_{2} /\right.$
\end{tabular}} \\
\hline & & Thermal & Hydro & Nuclear & Renewables & $\begin{array}{l}\text { (billion } \\
\text { kWhe) }\end{array}$ & Max & Min & Best \\
\hline \multirow{3}{*}{ Africa } & South Africa & 93 & 1 & 7 & 0 & 196 & 1.06 & 0.620 & 0.840 \\
\hline & Egipt & 81 & 19 & 0 & 0 & 72 & 0.998 & 0.543 & 0.770 \\
\hline & Average & 90 & 5 & 5 & 0 & 287 & 1.049 & 0.604 & 0.827 \\
\hline \multirow{3}{*}{$\begin{array}{c}\text { South } \\
\text { America }\end{array}$} & Argentina & 59 & 34 & 7 & 0 & 85 & 0.814 & 0.402 & 0.608 \\
\hline & Brazil & 6 & 89 & 1 & 4 & 339 & 0.440 & 0.056 & 0.248 \\
\hline & Average & 17 & 78 & 3 & 3 & 431 & 0.514 & 0.125 & 0.320 \\
\hline \multirow{4}{*}{$\begin{array}{c}\text { North } \\
\text { America }\end{array}$} & Canada & 28 & 58 & 13 & 1 & 566 & 0.562 & 0.198 & 0.380 \\
\hline & Mexico & 79 & 14 & 4 & 3 & 199 & 0.960 & 0.530 & 0.745 \\
\hline & USA & 71 & 6 & 21 & 2 & 3719 & 0.846 & 0.481 & 0.663 \\
\hline & Average & 66 & 13 & 19 & 2 & 4484 & 0.815 & 0.447 & 0.631 \\
\hline \multirow{8}{*}{ Europe } & Germany & 62 & 4 & 30 & 4 & 545 & 0.700 & 0.540 & 0.610 \\
\hline & Spain & 50 & 18 & 27 & 4 & 223 & 0.400 & 0.530 & 0.480 \\
\hline & France & 8 & 14 & 77 & 1 & 520 & 0.270 & 0.080 & 0.090 \\
\hline & Italy & 79 & 18 & 0 & 3 & 259 & 0.520 & 0.630 & 0.590 \\
\hline & United & 74 & 1 & 24 & 2 & 361 & 0.890 & 0.640 & 0.640 \\
\hline & Kingdom & & & & & & & & \\
\hline & Sweden & 4 & 51 & 43 & 2 & 153 & 0.230 & 0.040 & 0.040 \\
\hline & Average & 45 & 18 & 34 & 3 & 2382 & 0.510 & 0.130 & 0.470 \\
\hline \multirow{5}{*}{ Asia } & China & 82 & 17 & 1 & 0 & 1288 & 0.999 & 0.548 & 0.773 \\
\hline & India & 83 & 14 & 3 & 0 & 512 & 1.000 & 0.555 & 0.777 \\
\hline & Japan & 60 & 8 & 30 & 2 & 1037 & 0.729 & 0.405 & 0.567 \\
\hline & Russia & 66 & 19 & 15 & 0 & 835 & 0.835 & 0.447 & 0.641 \\
\hline & Average & 72 & 15 & 12 & 1 & 3788 & 0.888 & 0.487 & 0.688 \\
\hline \multirow{3}{*}{ Pacific } & Australia & 91 & 8 & 0 & 1 & 198 & 1.069 & 0.609 & 0.839 \\
\hline & New Zeland & 32 & 58 & 0 & 11 & 38 & 0.613 & 0.223 & 0.418 \\
\hline & Average & 81 & 16 & 0 & 2 & 236 & 0.996 & 0.547 & 0.772 \\
\hline
\end{tabular}

Table 1. $\alpha$ values: $\mathrm{CO}_{2}$ emissions from power plants. 


\begin{tabular}{|c|c|c|c|c|c|c|c|}
\hline Region & Application & System model & $\begin{array}{l}\text { Internal } \\
\text { Volume } \\
\text { Capacity }\end{array}$ & Refrig. & $\underset{(g)}{A m o u n t}$ & \begin{tabular}{|c|} 
Energy \\
Consumption \\
$(\mathrm{kWh} / \mathrm{month})$ \\
\end{tabular} & \begin{tabular}{|c|} 
Ambient \\
Temperature \\
$\left({ }^{\circ} \mathrm{C}\right)$ \\
\end{tabular} \\
\hline $\begin{array}{c}\text { North } \\
\text { America }\end{array}$ & Household & $\begin{array}{l}\text { Top Mounted } \\
\text { Side by Side } \\
\text { Chest Freezer } \\
\text { Vertical } \\
\text { Freezer } \\
\text { Glass Door } \\
\text { Merchandiser } \\
\text { Vending } \\
\text { Machine }\end{array}$ & $\begin{array}{l}600 \mathrm{lt} \\
800 \mathrm{lt} \\
500 \mathrm{lt} \\
500 \mathrm{lt} \\
600 \text { cans } \\
600 \text { cans }\end{array}$ & $\begin{array}{l}\text { HFC134a } \\
\text { HFC134a } \\
\text { HFC134a } \\
\text { HFC134a } \\
\text { HFC134a } \\
\text { HFC134a }\end{array}$ & $\begin{array}{l}110 \\
150 \\
150 \\
120 \\
\\
330 \\
\\
400\end{array}$ & $\begin{array}{l}40 \\
50 \\
45 \\
55 \\
\\
210 \\
\\
\\
300\end{array}$ & $\begin{array}{l}32 \\
32 \\
32 \\
32 \\
\\
32 \\
\\
\\
\end{array}$ \\
\hline Europe & \begin{tabular}{|c|} 
Household \\
Light Commercial \\
\end{tabular} & $\begin{array}{c}\text { Small } \\
\text { Refrigerator } \\
\text { Combined } \\
\text { Refrigerator } \\
\text { Vertical } \\
\text { Freezer } \\
\\
\text { Chest Freezer } \\
\text { Display Case }\end{array}$ & $\begin{array}{l}250 \mathrm{lt} \\
430 \mathrm{lt} \\
200 \mathrm{lt} \\
\\
200 \mathrm{lt} \\
550 \mathrm{lt}\end{array}$ & $\begin{array}{l}\text { HC600a } \\
\text { HC600a } \\
\text { HC600a } \\
\\
\text { HFC134a } \\
\text { HFC134a }\end{array}$ & $\begin{array}{l}30 \\
60 \\
40\end{array}$ & $\begin{array}{l}15 \\
30 \\
25\end{array}$ & $\begin{array}{l}25 \\
25 \\
25\end{array}$ \\
\hline Asia & \begin{tabular}{|c|} 
Household \\
Light Commercial \\
\end{tabular} & $\begin{array}{l}\text { Compact } \\
\text { Refrigerator } \\
\text { Combined } \\
\text { Refrigerator } \\
\\
\text { Vending } \\
\text { Machine } \\
\end{array}$ & $\begin{array}{c}80 \mathrm{lt} \\
350 \mathrm{lt} \\
600 \mathrm{cans}\end{array}$ & $\begin{array}{l}\text { HC600a } \\
\text { HC600a } \\
\text { HFC134a }\end{array}$ & $\begin{array}{l}25 \\
50\end{array}$ & $\begin{array}{l}20 \\
25\end{array}$ & $\begin{array}{l}25 \\
25\end{array}$ \\
\hline
\end{tabular}

Table 2. Typical refrigeration equipment per market region.

Figure 5 clearly shows the strong influence of indirect effect due to energy consumption on the total $\mathrm{CO}_{2}$ emissions. In the North American market the direct contribution in the household applications ranges between 16 and 23\%, in light commercial between 11 and $12 \%$. In Europe, due to the use of hydrocarbons as refrigerants in household applications, direct contribution has practically no contribution to the TEWI. In the light commercial applications the direct contribution ranges between 2 and 25\%, with the higher values associated to the refrigerant fluid with higher GWP. The situation in Asia for household applications is very similar to Europe due to the use of hydrocarbons. Light commercial applications in Asia show a direct contribution of $10 \%$.

Therefore regardless of the market region and type of refrigerant system, indirect effect to global warming always represents the prevalent contribution to global warming.

From the previous data a sensitivity analysis can be carried out employing two different scenarios. In the first one the refrigerants were replaced by a new refrigerant with a low GWP, for example 1 and the energy consumptions was held constant. 


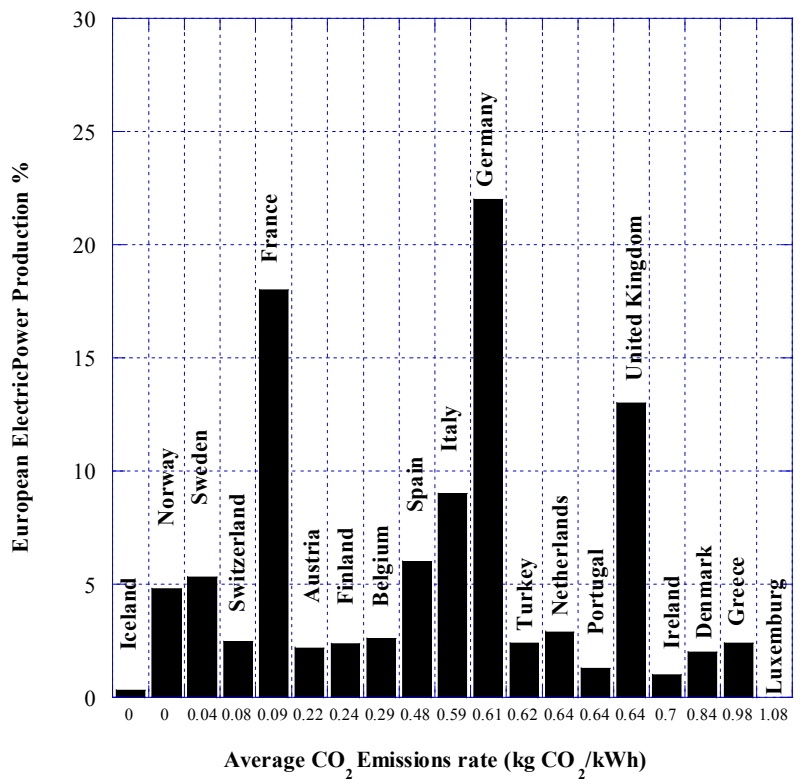

Figure 3. Average power plant emission rates and electricity production for European nations.
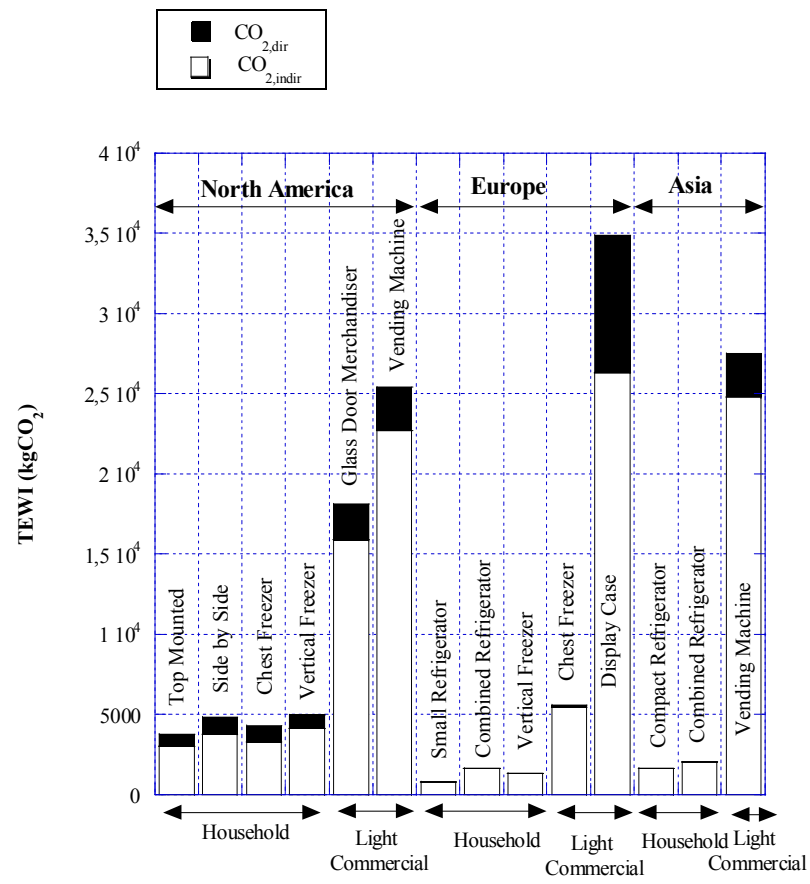

Figure 4. TEWI values for different application and different market region. 


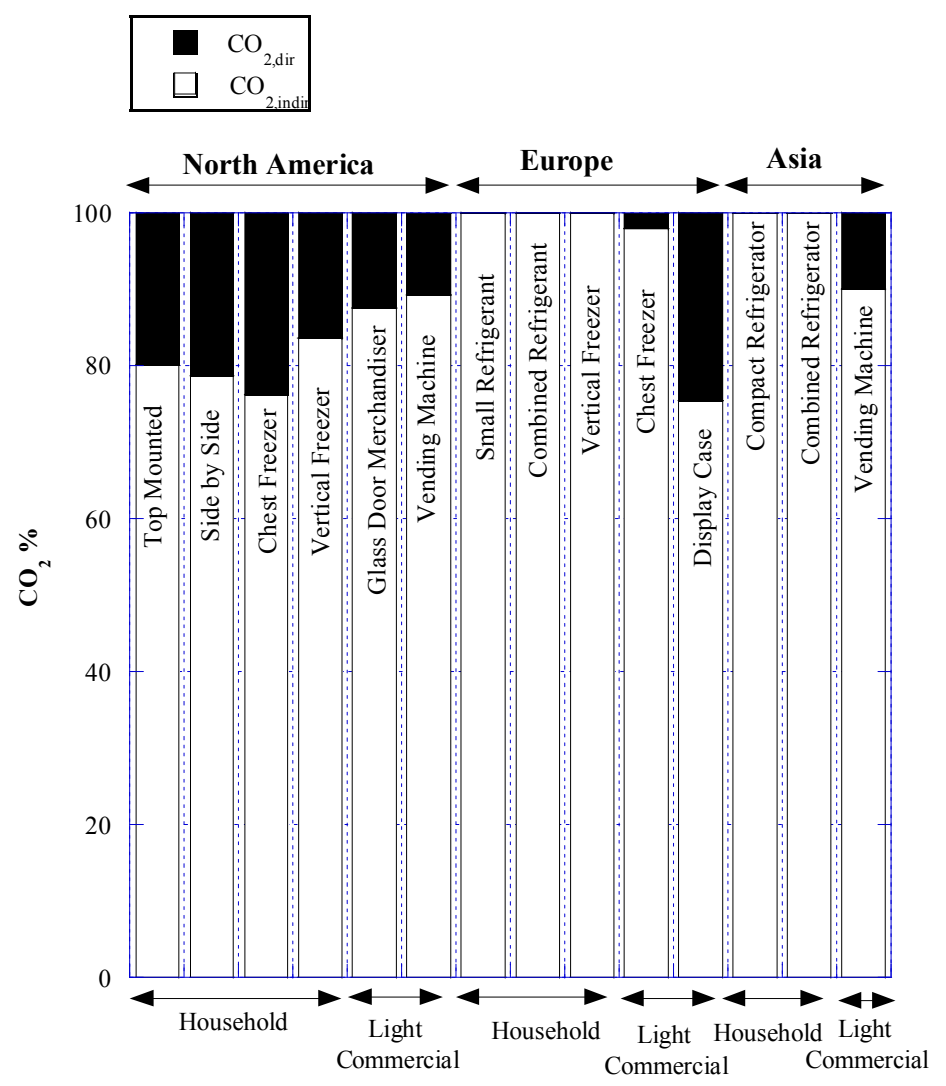

Figure 5. Direct and indirect $\mathrm{CO}_{2}$ emissions percentage for different application.

In Figure 6 are reports the results of the first scenario in terms of $\triangle$ TEWI/TEWI.

The Figure 6 clearly shows that in the North America market the replacement of the refrigerant fluid with a unitary GWP refrigerant decreases the TEWI from -24 to $-16 \%$ in household application, from-12 to $-11 \%$ in light commercial applications. In the European and Asian market, due to current use of hydrocarbons in household applications, the replacement of the refrigerant fluid does not provide any additional benefit. Whereas in the light commercial application in the European market the TEWI decreases between -24 and $-2 \%$, in the Asian market decreases of about $-10 \%$.

A second scenario the energy consumption was reduced by $30 \%$ and the refrigerant fluid was not changed. In Figure 7 are reports the results of the first scenario in terms of $\triangle$ TEWI/TEWI. 


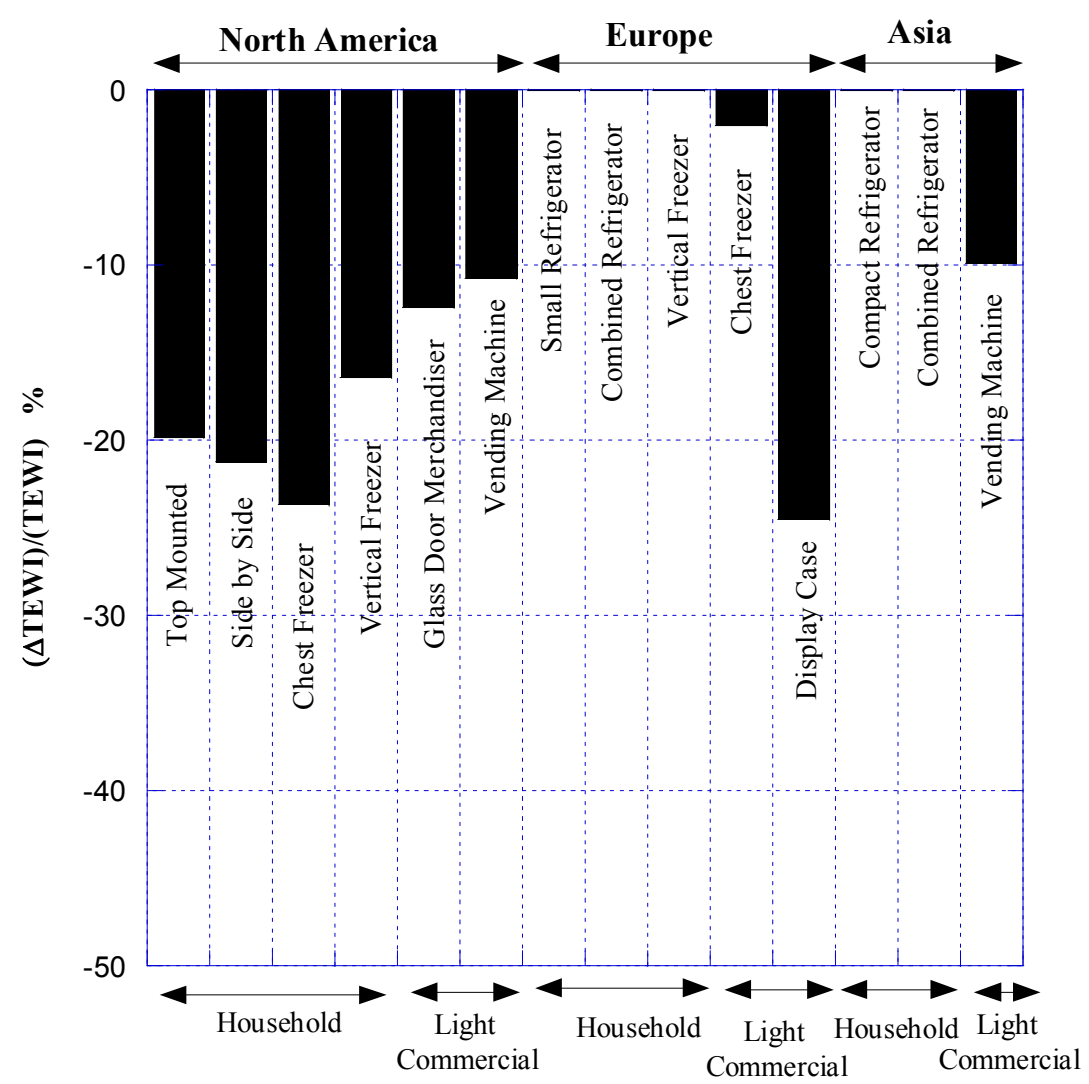

Figure 6. $\triangle \mathrm{TEWI} / \mathrm{TEWI}$ replacing the refrigerant fluid with a low GWP fluid.

The figure clearly shows that improving the energy efficiency by $30 \%$, in the North America Market the TEWI decreases between -47 and $-38 \%$, in the European market between -47 and $-30 \%$, in the Asian market between -37 and $-30 \%$.

The previous analysis clearly shows that the indirect effect on TEWI is stronger than the direct one in the household and light commercial applications regardless of the market region. Therefore it seems more effective, in order to decrease the global warming impact of a refrigeration system, to focus the attention on the improvement of the energy efficiency. To this aim the replacement of a refrigerant fluid with one with low GWP but that introduces penalties to the equipment efficiency, must be avoided. 


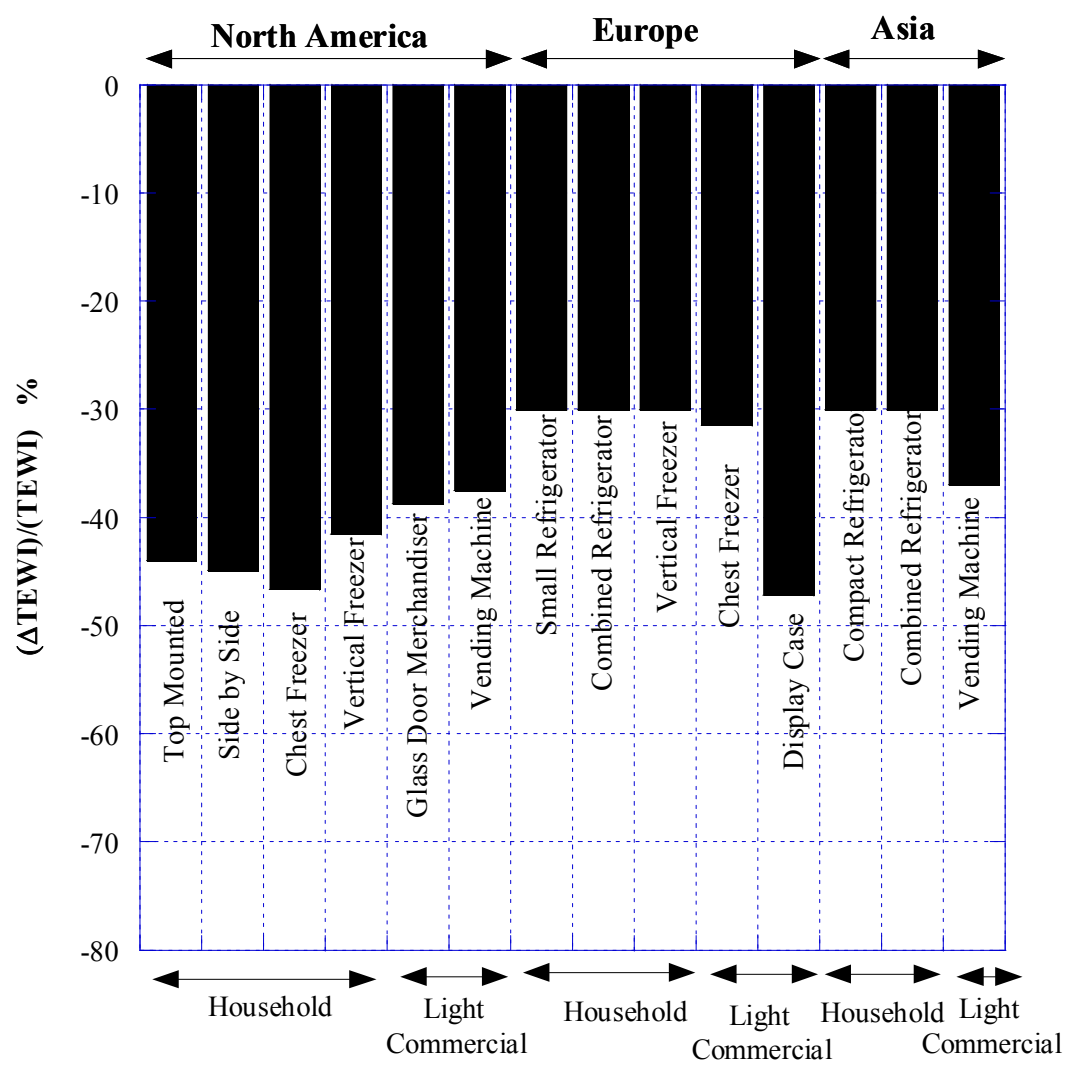

Figure 7. $\triangle \mathrm{TEWI} / \mathrm{TEWI}$ decreasing energy consumption by $30 \%$.

\section{An experimental evaluation of the greenhouse effect in R22 substitution with R407C}

\subsection{R22 substitution with R407C}

R22 is an HCFC with an ODP of 0.05 and a GWP of 1700 and is the most widely used refrigerant today in commercial, domestic and industrial applications, and its phase-out will impact a large number of plants in the world. Therefore retrofitting these systems would alleviate the R22 phase-out problem. This opportunity could be cheaper than the installation of new plants, especially for supermarkets, data centers, factories and hospitals. Refrigerant replacement candidates have been checked for environmental and safety requirements, compatibility with lubricant oil, filters, and sealing. In order to establish the best substitute in a specified system among the candidates, it is necessary to estimate energetic performances after refrigerant replacement. In the last years, many companies have expended much effort to develop and characterize refrigerants able to increase the energetic efficiency of a refrigeration system, depending on its application. Similarly many 
researchers have investigated the energetic performances of the newest substitutes of R22 $[27,28]$.

During this transition period, many industrial and commercial applications used R407C to retrofit R22. The latter is the only drop-in substitute, and is an HFC with zero ODP and a GWP (1700) lower than that of R22. R407C is a zeotropic mixture of R32/R125/R134A (23/25/52\% in weight).

Even if R407C has thermo-physical properties similar to those of R22 and it is nonflammable and non-toxic, it is incompatible with mineral or alkyl-benzene oil. Consequently, the R22 retrofit with R407C implies the adoption of polyester oil.

In the preset paper an experimental comparison between TEWI of R22 and R407C has been carried with a vapour compression pilot plant [29].

\subsection{The experimental apparatus}

The experimental apparatus is reported in Figure 8 and consists of three loops: the refrigerant loop, the condensing water loop and the evaporating water-glycol loop [30].

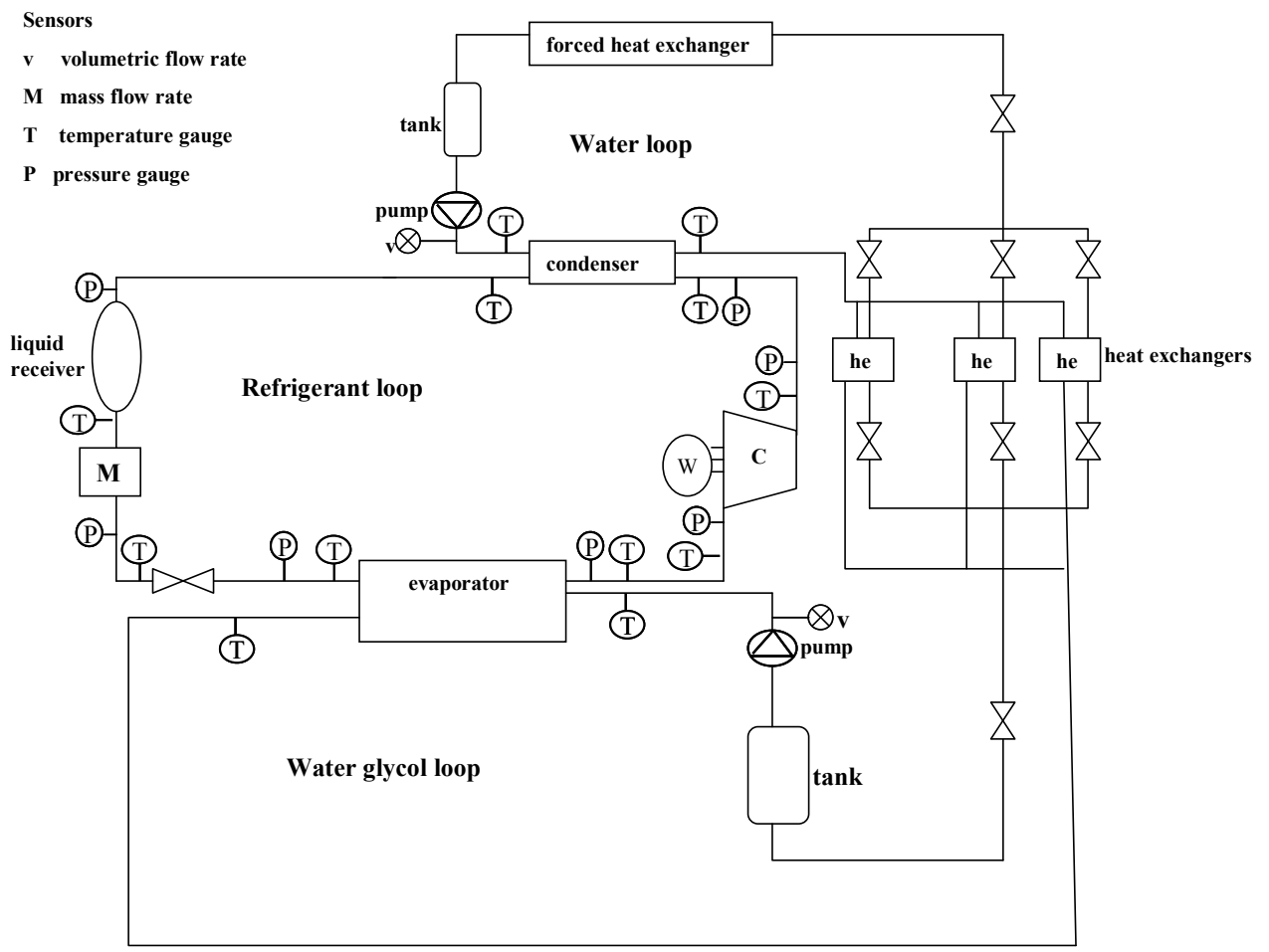

Figure 8. Flow-sheet of the experimental apparatus. 
The refrigerant loop is a vapour compression plant consisting in a semihermetic compressor, a plate condenser connected to a liquid receiver, a thermostatic valve and a plate evaporator.

The water loop is used for cooling the refrigerant flowing in the plant. It consists in the condenser, a circulation pump, an inertial tank, an air operated cooling exchanger and three plate heat exchangers. The refrigerant duty consists in a water-glycol mixture $(70 / 30 \%$ in weight) continuously heated in the three intermediate plate heat exchangers by means of the water condensing loop. Whenever required, additional heating is provided by three electrical resistances inserted into an inertial tank (maximum power $12 \mathrm{~kW}$ ). The waterglycol loop consists in the evaporator, a circulation pump and an inertial tank.

In the main loop measurements are performed of: the pressure and $\mathrm{f}$ the temperature at the inlet and at the outlet of each device, the mass-flow rate at the outlet of the liquid receiver and the electrical power supplied to the compressor. As regards the water-glycol loop, the temperature at the inlet and outlet of the evaporator, at the intermediate heat exchangers, and at the inertial tank are determined. Measurements are carried out of the volumetric flowrate at the inlet of the tank and also of the electrical power supplied to the resistances. The secondary loop at the condensed has been instrumented in a similarly way. A detailed indication of sensor position is provided in Figure 8.

Pressures are measured by piezoelectric transducers $(\mathrm{P})$ in the pressure ranges are $0-7$ bar and $0-30$ bar with an accuracy of $\pm 0.5 \%$ F.S. Temperature are measured by means of fourwire $100 \Omega$ platinum resistance thermometers $(\mathrm{T})$ with an accuracy of $\pm 0.15 \mathrm{~K}$. The electric power input to the compressor and that required by the electrical resistances are measured by a Watt transducer with an accuracy of $\pm 0.2 \%$. Refrigerant mass flow rate is measured by a Coriolis effect mass flow-meter $(\mathrm{M})$ with an accuracy of $\pm 0.2 \%$.

Two turbine flow-meters are employed for measuring the condensing water volumetric flow rate (v) and that of the water-glycol mixture circulating in the evaporator (v) with an accuracy of $\pm 0.25 \%$.

According to Tables 3 and 4, different experimental situations are determined by varying the operating conditions.

Table 3 reports $\mathrm{T}_{\mathrm{w}, \text { in,co }} / \mathrm{T}_{\mathrm{w}, \text { out,co }}$ (the inlet and the outlet temperature at the condenser water side) and $\mathrm{TMT}_{\mathrm{wg}}$ (the mean thermodynamic temperature of the water glycol mixture). Table 3 reports three different set of runs. In each set of runs, the values of the inlet and outlet temperature at the condenser water side have been kept constant, whereas TMTwg has been varied. The tests conducted at $30 / 35$ and $35 / 40{ }^{\circ} \mathrm{C}$ refer to a refrigeration plant, whereas the tests at $45 / 50{ }^{\circ} \mathrm{C}$ refer to a heat pump.

Table 4 reports TMTwg and Tco, i.e. the condensing temperature of the refrigerant fluid (as regards $\mathrm{R} 407 \mathrm{C}$, that is an azeotropic mixture, a mean temperature between dew and bubble point was considered). Table 4 reports three different sets of run. In each set the values of $\mathrm{TMT}_{w g}$ and the refrigerant duty has been kept constant, whereas Tco has been varied. 


\begin{tabular}{|c|cc|}
\hline \multicolumn{2}{|c|}{ Evaporator } & \multicolumn{2}{|c|}{ Condenser } \\
\hline TMTwg $\left({ }^{\circ} \mathbf{C}\right)$ & Tout, $\mathbf{w}$ & Tin, $\mathbf{w}\left({ }^{\circ} \mathbf{C}\right)$ \\
\hline-7.9 & & \\
-4.9 & & \\
6.2 & 35 & 30 \\
9.8 & & \\
13.2 & & \\
\hline-5.7 & & \\
-5.2 & & \\
7.5 & 40 & 35 \\
10.2 & & \\
12.1 & & \\
\hline 7.2 & & \\
10.3 & & \\
12.1 & & \\
14.3 & & \\
\hline
\end{tabular}

Table 3. The different operating conditions.

\begin{tabular}{|l|l|}
\hline Condenser & Evaporator \\
\hline Tco $\left({ }^{\circ} \mathbf{C}\right)$ & TMT $_{w g}\left({ }^{\circ} \mathbf{C}\right)$ \\
\hline $41.8-42.1-44.7-45.9-47.3-49.4-50.4-52.9$ & 8 \\
\hline $42.7-44.2-44.8-45.9-46.5-48.3-51.7-53.3$ & 10 \\
\hline $43.7-44.0-44.6-46.0-47.2-48.0-50.2-53.0$ & 13 \\
\hline
\end{tabular}

Table 4. The different operating conditions

Table 5 reports the parameters adopted for the TEWI evaluations.

\begin{tabular}{|l|l|}
\hline Parameter & Value \\
\hline $\mathrm{H}$ & $950 \mathrm{~h} /$ year \\
\hline $\mathrm{PL}$ & $5 \% /$ year \\
\hline $\mathrm{PR}$ & $25 \%$ \\
\hline $\mathrm{V}$ & 10 years \\
\hline$\alpha$ & $0.6 \mathrm{~kg} \mathrm{CO} / \mathrm{kWhe}$ \\
\hline
\end{tabular}

Table 5. Parameters in TEWI evaluation. 


\subsection{Results and discussion}

\subsubsection{The direct contribution}

This contribution has been evaluated, referring to the experimental plant, on the basis of the measured charge of the plant pertaining to R22 and to R407C and by assuming refrigerant leaks in terms of fraction of refrigerant charge.

The direct contributions to the greenhouse effect to R22 and to R407C during the plant useful life corresponds to the same net impact on global warming as the release of 6375 and $5400 \mathrm{~kg}$ of carbon dioxide, respectively.

The R22 direct contribution is about $15 \%$ greater than the R407C one, a direct consequence of the greater GWP and charge pertaining to R22.

\subsubsection{The indirect contribution}

The thermodynamic parameter that affects this contribution is the coefficient of performance of the plant. In the following Figs 9-11, is shown the behaviour of the COP for different experimental situations. Those figures are referred to the test conditions pertaining to different inlet and outlet water temperatures in the condenser $(30 / 35,35 / 40$, $45 / 50{ }^{\circ} \mathrm{C}$ ), and then to different refrigerant condensing temperatures. The first two diagrams refer to a refrigerant plant, whereas the third diagram accounts for a heat pump. In all instances, COP increases with water glycol mean thermodynamic temperature. Indeed, with increasing the latter, both the refrigerating duty and the mechanical power consumption at the compressor increase. The relative increase of the former, however, exceeds that of the latter. As a consequence, COP increases. For all the experimental runs conducted, the performance coefficient of R407C is lower than that pertaining to R22. Therefore, the indirect contribution to the greenhouse effect of $\mathrm{R} 407 \mathrm{C}$ is always higher than of R22.

The broken line reported in the previous diagrams estimates a theoretical COP value for R407C (COP407C ${ }^{*}$ ) that might compensate for the higher direct contribution of R22 to the greenhouse effect for this plant in the same operating conditions.

Indeed, if the R407C $\mathrm{COP}^{*}$ were about 5\% lower than that or R22, the greater direct contribution of the latter would be balanced by employing the former in the summer cycle (refrigeration). Unfortunately, as shown in figure 9 and 10, the actual value of the COP pertaining to $\mathrm{R} 407 \mathrm{C}$ is lower than that corresponding to this theoretical value (COP407C*). Therefore, under these experimental conditions, R407C has a greater greenhouse effect than R22. At this stage, however, the difference can not be determined quantitatively. In runs performed with water inlet and outlet temperature of 30 and $35^{\circ} \mathrm{C}$, respectively, the COP of $\mathrm{R} 407 \mathrm{C}$ is lower than that pertaining to $\mathrm{R} 22$ by $8-11 \%$. In runs with water inlet and outlet temperature of 35 and $340{ }^{\circ} \mathrm{C}$, respectively, the COP of $\mathrm{R} 407 \mathrm{C}$ is lower by $16-19 \%$. Therefore, the difference between the actual COP value and $\mathrm{COP}^{*}$ of $\mathrm{R} 407 \mathrm{C}$ is remarkable, especially in the $35 / 40{ }^{\circ} \mathrm{C}$ runs. 
On the contrary, the best performance is achieved with inlet and outlet water temperature of 45 and $50{ }^{\circ} \mathrm{C}$, respectively, corresponding to a winter cycle (heat pump). In this case, the $\mathrm{COP}^{*}$ pertaining to $\mathrm{R} 407 \mathrm{C}$ that might compensate the higher direct contribution of $\mathrm{R} 22$ were about 3\% lower than that of R22. Indeed, the actual COP value of R407C is always about $3.2 \%$ lower than that pertaining to R22, except for the run at TMTwg $=7.4{ }^{\circ} \mathrm{C}$. In this case, it is $6.6 \%$ lower. The difference between the actual COP value and COP* of R407C is in this case lower.

The less satisfactory situation corresponds to the run with water inlet and outlet temperature of 35 and $40^{\circ} \mathrm{C}$, respectively.

A set of runs have been conducted by keeping the mean thermodynamic water glycol temperature and the refrigerant duty constant and by varying the condensation temperature of the refrigerant fluids in order to better clarify the reason of this behaviour.

The results of the runs obtained with TMTwg $=10^{\circ} \mathrm{C}$ are summarized in figure 12. Similar results are obtained in all the experimental runs performed with other water glycol mean temperatures reported in Table 5.

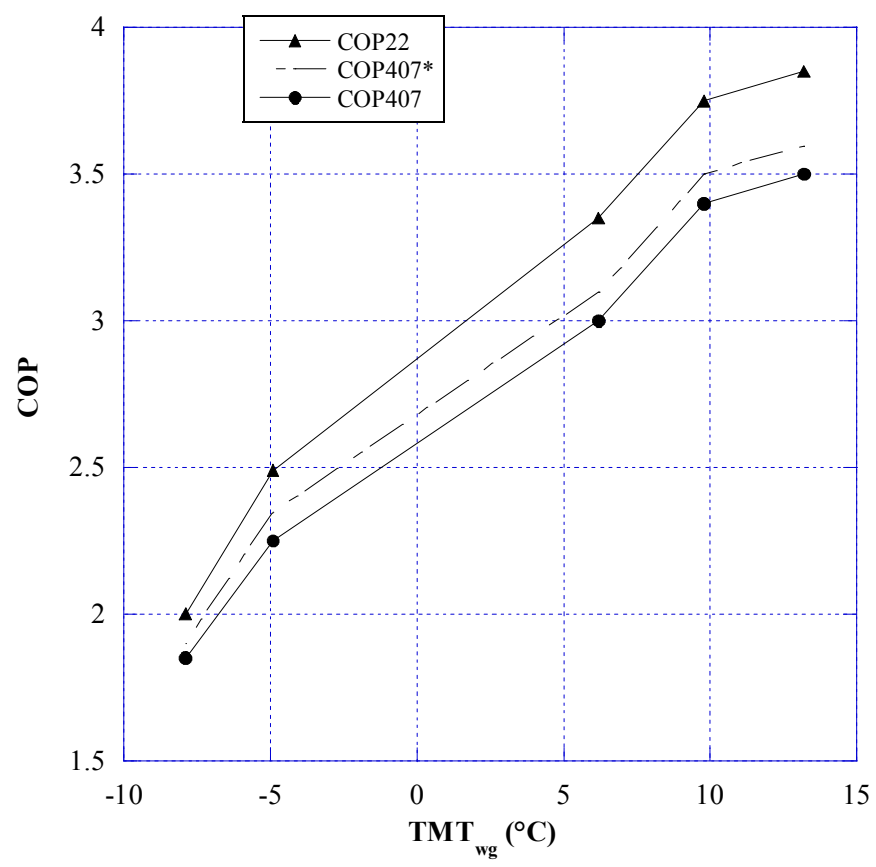

Figure 9. COP vs mean thermodynamic temperature of water-glycol mixture, Tin, $\mathrm{w}, \mathrm{co}=30^{\circ} \mathrm{C}$, Tout $, \mathrm{w}, \mathrm{co}=35^{\circ} \mathrm{C}$. 
For both fluids, the COP decreases with the condensation temperature. The shape of the two curves, however, is such that the maximum COP difference is achieved at intermediate value of the condensation temperatures $\left(46-48^{\circ} \mathrm{C}\right)$. The value of the condensation temperature pertaining to the corresponding run performed with water inlet and outlet temperatures of $35 / 40^{\circ} \mathrm{C}$ fall in this range.

The general behaviour of the experimental results can be explained in the light of the dependence of the compressor duty on the compression ratio. The compressor duty increases nonlinearly with the compression ratio. The compression ratio increases with the condensing temperature at equal mean thermodynamic temperature and, therefore, at equal evaporating pressure. Therefore, COP decreases with increasing compression duty at equal refrigerant duty.

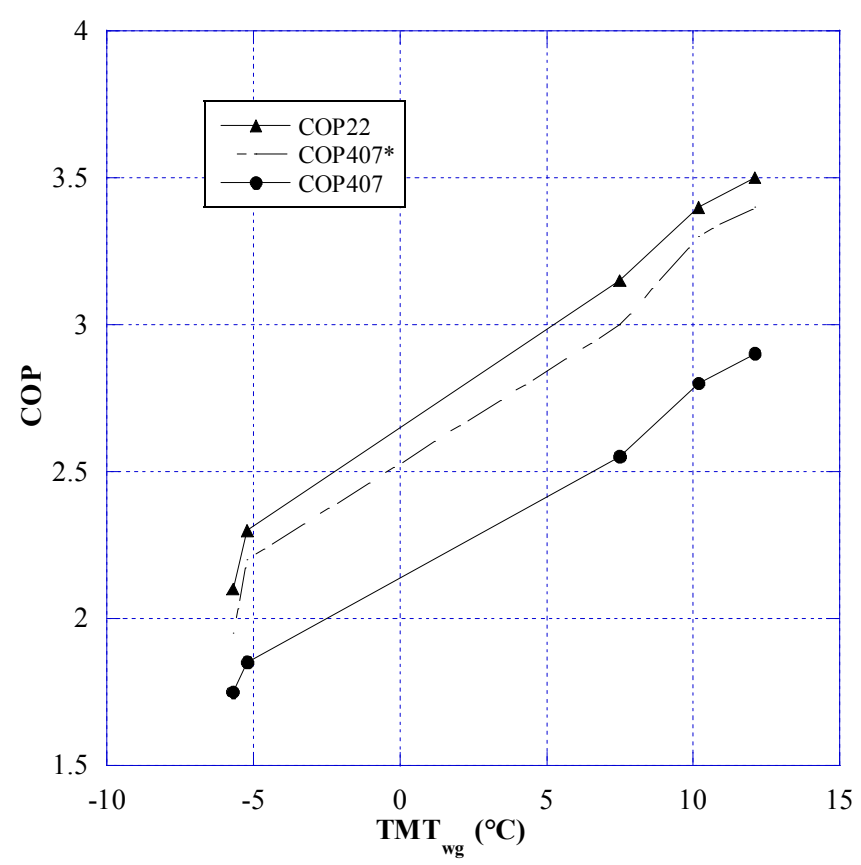

Figure 10. $\mathrm{COP}$ vs mean thermodynamic temperature of water-glycol mixture, $\mathrm{Tin}, \mathrm{w}, \mathrm{co}=35^{\circ} \mathrm{C}$, Tout, $\mathrm{w}, \mathrm{co}=40^{\circ} \mathrm{C}$. 


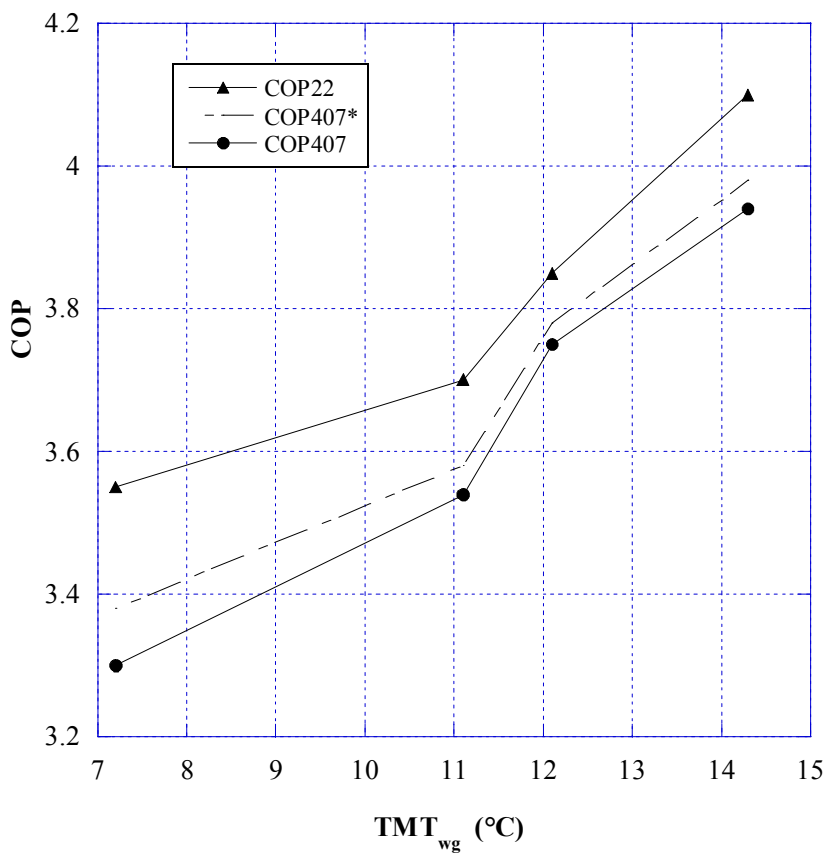

Figure 11. COP vs mean thermodynamic temperature of water-glycol mixture, Tin, $\mathrm{w}, \mathrm{co}=40^{\circ} \mathrm{C}$, Tout, $\mathrm{w}, \mathrm{CO}=45^{\circ} \mathrm{C}$.

\subsubsection{Overall evaluation}

The TEWI has been evaluated as a function of the mean thermodynamic temperature of the water glycol mixture at the evaporator.

The results are summarised in Figs 13-15.

According to the previous discussion, in Figs. 13 and 14, the TEWI pertaining to R407C is greater than that corresponding to R22. The difference ranges from a minimum of 2.3 to a maximum of $4.3 \%$ in the runs performed at $30 / 35^{\circ} \mathrm{C}$. The effect is even more marked in the runs performed at $35 / 40{ }^{\circ} \mathrm{C}$ (about $11 \%$ ). This yields an increased $\mathrm{CO} 2$ emission of about 320 $\mathrm{kg} /$ year for the plant under consideration when working as a refrigerator with R407C as compared to R22.

On the contrary, the two fluids are virtually equivalent in terms of greenhouse effect, when the plant acts as a heat pump in the winter cycle $\left(40 / 45^{\circ} \mathrm{C}\right.$ runs, Fig. 15). 


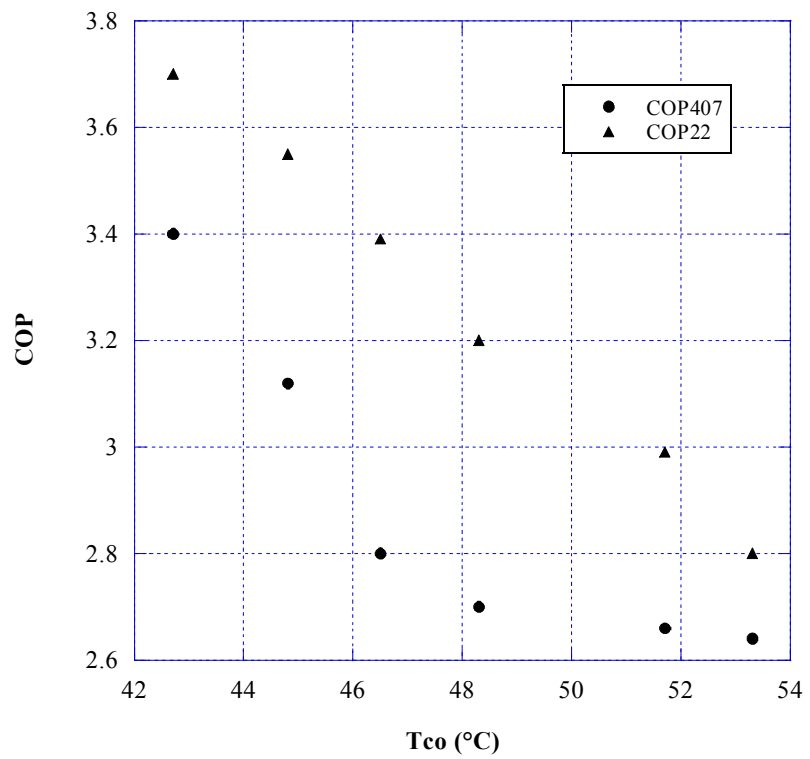

Figure 12. COP vs condensing temperature of the refrigerant fluid.

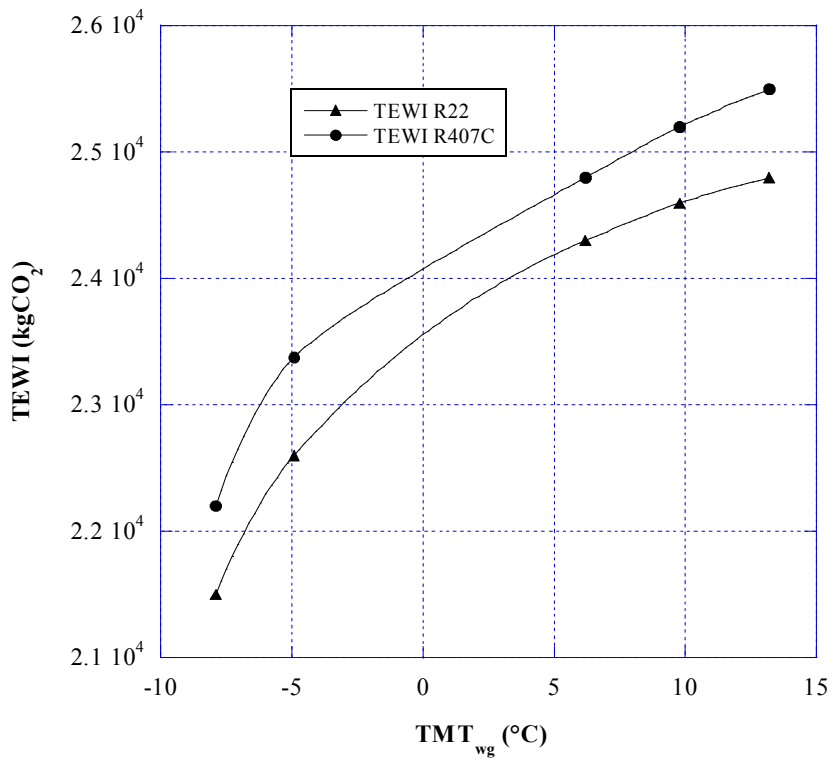

Figure 13. TEWI vs mean thermodynamic temperature of water-glycol mixture, $\mathrm{Tin}, \mathrm{w}, \mathrm{CO}=30^{\circ} \mathrm{C}$, Tout, $\mathrm{w}, \mathrm{CO}=35^{\circ} \mathrm{C}$. 


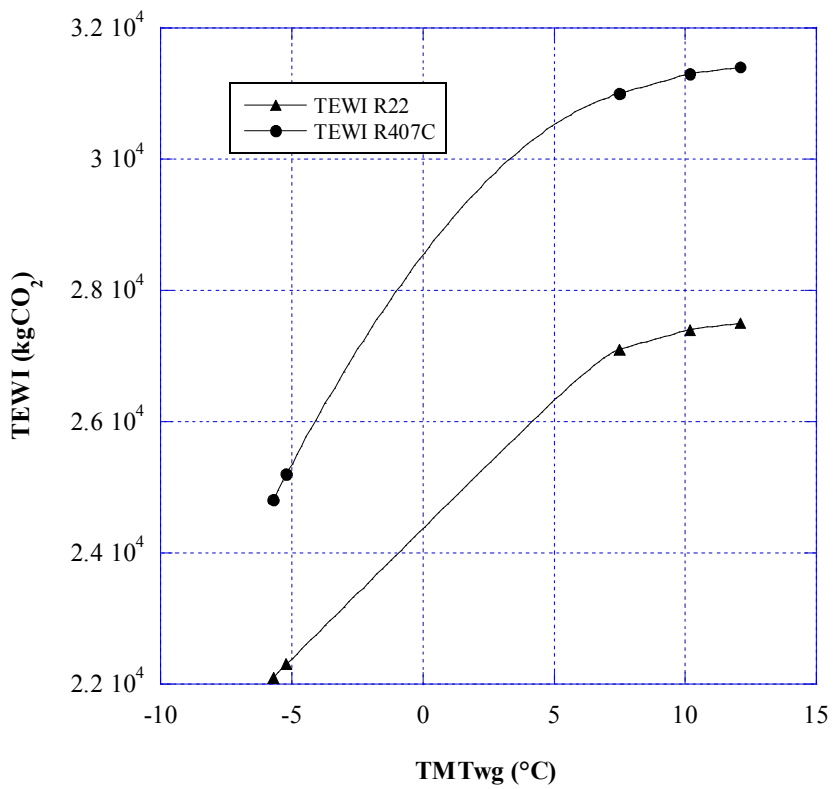

Figure 14. TEWI vs mean thermodynamic temperature of water-glycol mixture, $\operatorname{Tin}, \mathrm{w}, \mathrm{co}=35^{\circ} \mathrm{C}$, Tout, $\mathrm{w}, \mathrm{CO}=40^{\circ} \mathrm{C}$.

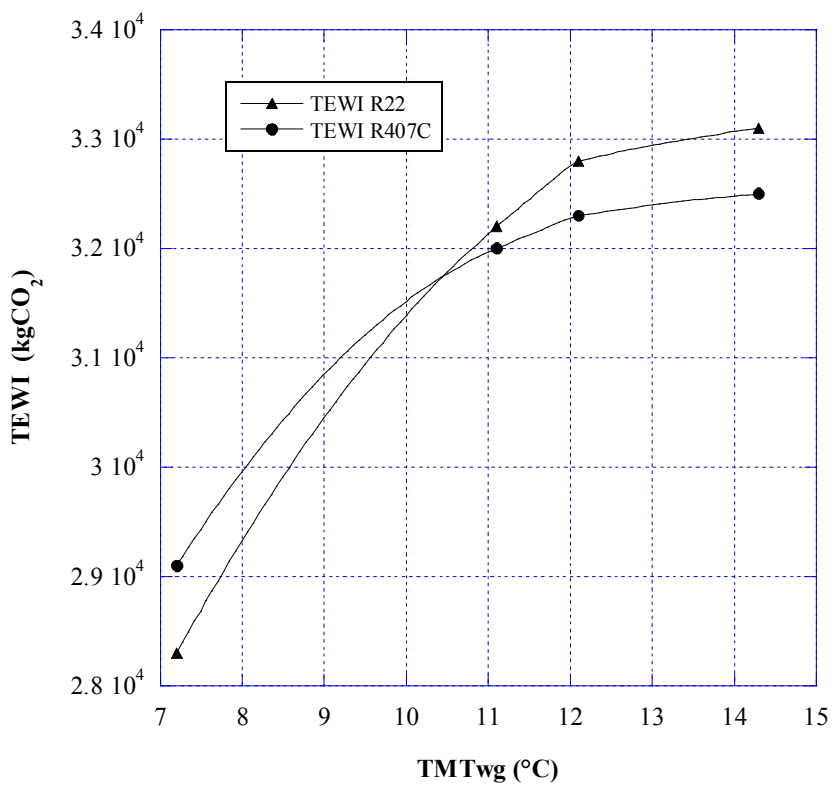

Figure 15. TEWI vs mean thermodynamic temperature of water-glycol mixture, Tin, $\mathrm{w}, \mathrm{co}=40^{\circ} \mathrm{C}$, Tout, $\mathrm{w}, \mathrm{co}=45^{\circ} \mathrm{C}$. 


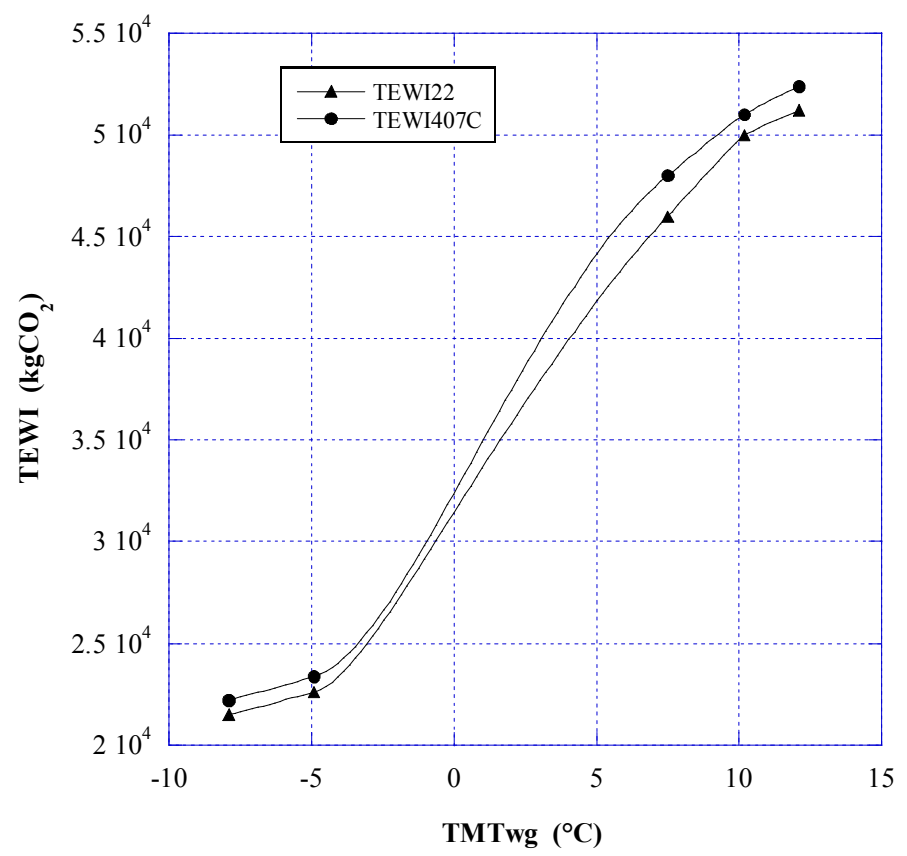

Figure 16. TEWI vs mean thermodynamic temperature of water-glycol mixture for the reversible pump working for the whole year.

Figure 16 reports the overall results for the reversible heat pump working for the whole year (both in winter and summer cycle). It can be seen that the TEWI pertaining to R407C is slightly larger than that of R22 (2.7-4.7\%). This corresponds to an increased $\mathrm{CO}_{2}$ production of about $87 \mathrm{~kg} /$ year.

In Figure 17, the TEWI difference ( $\triangle \mathrm{TEWI}$ ) between the two fluids is reported as a function of the condensation temperature for three different values of mean thermodynamics temperature of the refrigerant duty $\left(810,13{ }^{\circ} \mathrm{C}\right.$ respectively). The $\triangle T E W I$ attains a maximum in the intermediate range of condensation temperatures. On the contrary, for high condensation temperature, $\triangle$ TEWI becomes negative. In the condensation temperature range explored, the $\triangle \mathrm{TEWI}$ decreases with increasing mean thermodynamic temperature of the refrigerant duty.

\subsection{Concluding remarks}

The problem of R22 substitution with R407C in terms of global warming effect has been examined. R407C is harmless as far as ozone is concerned. It yields, however, a negative contribution to the greenhouse effect, both directly with a GWP of 1600 and indirectly, because of the lower energetic efficiency that results in higher $\mathrm{CO}_{2}$ emissions.

Experimental readings have been carried out on a vapour compression pilot plant. The contribution to the greenhouse effect has been taken account by evaluating the TEWI values. 
The analysis of the experimental runs leads to the conclusion that the R22 direct contribution to the greenhouse effect is greater than that pertaining to R407C (+15\%). The COP corresponding to $\mathrm{R} 407 \mathrm{C}$ is $3.3-19 \%$ lower than that pertaining to R22. The indirect contribution of the former is, therefore, always higher than that of the latter. Therefore, the final result depends on the operating conditions adopted in the experimental runs.

The most unfavourable conditions are reached for the water inlet and outlet temperature at the condenser of $35-40{ }^{\circ} \mathrm{C}$, respectively. This situation corresponds to a condensation temperature in the range $43-47^{\circ} \mathrm{C}$ and to an evaporation temperature in the range $-12 /-7^{\circ} \mathrm{C}$. Under these conditions, the TEWI pertaining to R407C exceeds that of R22 by about $11 \%$. Therefore, the substitution of R22 with R407C should be unacceptable in this specific situation if specific reference is made to the greenhouse effect.

On the contrary, the most favourable conditions are met for the water inlet and outlet temperature at the condenser of $45-50{ }^{\circ} \mathrm{C}$, respectively. This situation corresponds to a condensation temperature in the range $53-58{ }^{\circ} \mathrm{C}$ and to an evaporation temperature inlet range $2-10{ }^{\circ} \mathrm{C}$. In this case, the two fluids behave in a similar way as regards the greenhouse effect.

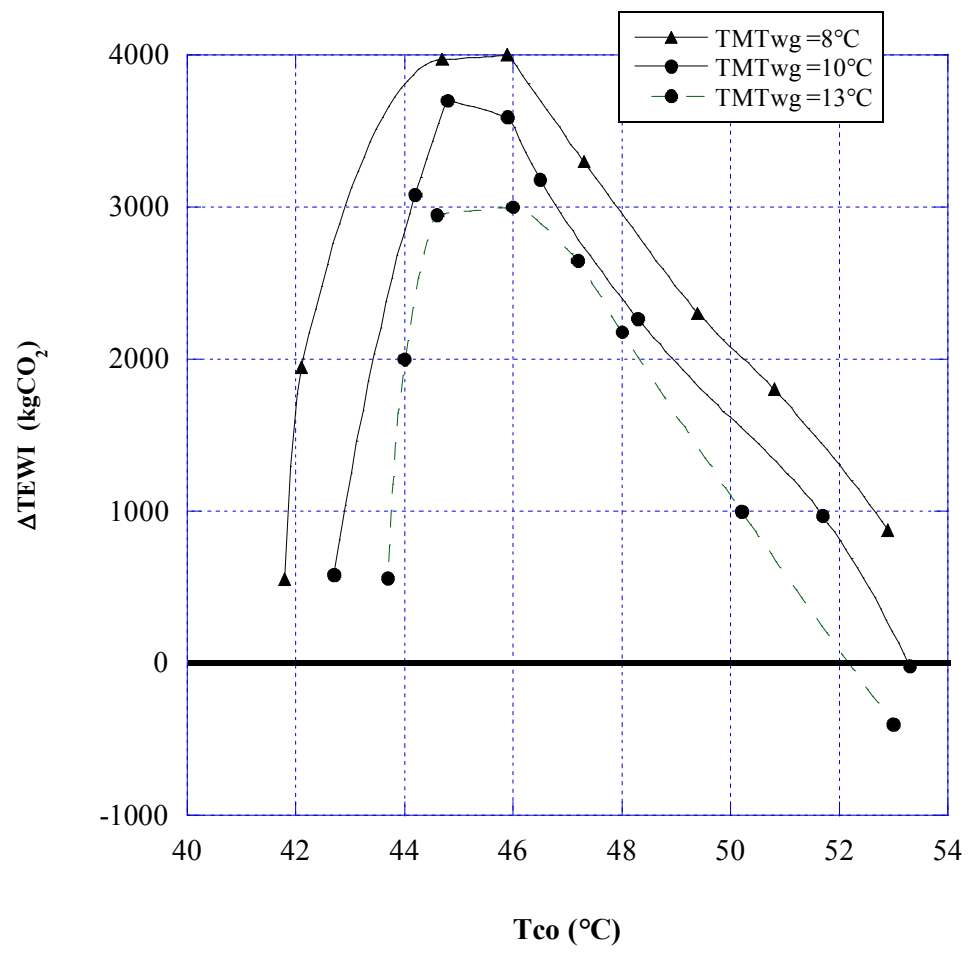

Figure 17. Difference between R407C and R22 TEWI vs condensing temperature of the refrigerant fluid for three different thermodynamic temperature of water-glycol mixture $\left(8,10\right.$ and $\left.13{ }^{\circ} \mathrm{C}\right)$. 
For high evaporation temperatures, the TEWI of R407C is even slightly lower than that of R22. As a consequence, the substitution of R22 with R407C is favourable, since no harm is produced to the ozone layer and no increase in $\mathrm{CO}_{2}$ emission is made. As a final remark the substitution of R22 with R407C is convenient from the point of view of the greenhouse effect for high condensation (over $50^{\circ} \mathrm{C}$ ) and evaporation temperatures of the refrigerant fluid.

\section{An experimental evaluation of the greenhouse effect in R22 substitution with R422D}

\subsection{R22 substitution with R422D}

As aforementioned, R22 is the most widely used refrigerant today in commercial, domestic and industrial applications, and its phase-out will impact a large number of plants in the world. Therefore retrofitting these systems would alleviate the R22 phase-out problem. This opportunity could be cheaper than the installation of new plants, especially for supermarkets, data centers, factories and hospitals. Refrigerant replacement candidates have been checked for environmental and safety requirements, compatibility with lubricant oil, filters, and sealing. In order to establish the best substitute in a specified system among the candidates, it is necessary to estimate energetic performances after refrigerant replacement. In the last years, many companies have expended much effort to develop and characterize refrigerants able to increase the energetic efficiency of a refrigeration system, depending on its application.. Even if R407C has thermo-physical properties similar to those of R22 and it is non-flammable and non-toxic, it is incompatible with mineral or alkyl-benzene oil. Consequently, the R22 retrofit with R407C implies the adoption of polyester oil, which requires a difficult and expensive conversion. Furthermore, in comparison with R22, experimental tests carried out with R407C have pointed out a reduction in the energetic performances. Recent additions to the aforementioned alternative refrigerants for R22 are R422A, R422B, R422C and R422D. The U.S. environment protection agency reported these alternative refrigerants could be used for household and light commercial air conditioning applications. In particular, R422D is a zeotropic mixture of R134a/ R125/ R600a (31.5/65.1/3.4\% in weight) with no ODP and a GWP of 2230. This refrigerant fluid, originally was designed to replace R-22 in existing direct expansion water chiller systems. It can also be used in residential and commercial air conditioning and medium-temperature (and low) refrigeration systems. Minor equipment modifications (replacement of the filter drier and elastomeric seals/gaskets that are exposed to refrigerant, refill of oil if required) or components tuning may be required in some applications. It is also compatible with mineral oil and there is no need to replace it with synthetic oil. Field experience has shown that R422D provides performance that meets customer requirements in most retrofitted systems. It provides similar cooling capacity to R22 and it is capable to operate at significantly lower compressor discharge temperature.

In this scenario, it is seems sensitive to inquire what is the actual impact of R422D on the environment, when it is employed in retrofitted R22 devices. It is well known that the GWP 
of R422D is higher than that of R22, but not much is known about the energy efficiency of systems retrofitted by R422D. For this purpose, a comparison of the energy consumptions of R22 and R422D for a direct expansion refrigerator applied to a commercial cold store is proposed. The experimental investigation has been carried out for different application conditions: medium temperature refrigeration for meat, fish, and dairy cases and high temperature refrigeration for air conditioning and cooling of preparation room. Subsequently, we investigated into the possibility of reducing the environmental impact of retrofitting R22 systems with R422D by means of a sensitivity analysis of some of the functional parameters.

\subsection{Experimental facility}

The experimental vapor compression refrigeration plant, applied to a commercially available cold store as shown in Fig. 18, consists of a semi-hermetic reciprocating compressor, an air condenser followed by a liquid receiver, a R22 mechanic thermostatic expansion valve to feed an air-cooled evaporator inside the cold store.

The compressor, as declared by the manufacturer, can operate with the fluid R22 and it is lubricated with mineral oil. With an evaporation temperature range between -20 to $10^{\circ} \mathrm{C}$, a $35^{\circ} \mathrm{C}$ condensing temperature, and utilizing $\mathrm{R} 22$ at the nominal frequency of $50 \mathrm{~Hz}$, the compressor refrigerating capacity is in the range of 1.4-4.4 kW. A blower drives the airflow through a thermally insulated channel where some electrical resistances are located with the objective of controlling the temperature of the airflow crossing the condenser. To fix the temperature of the airflow in accordance with the values sought, we changed the voltage supply of the electrical resistances by means of a PID controller. The cooling load in the cold store is emulated by means of additional electrical heaters wired to a voltage regulator. To keep the air temperature reasonably constant in the cold store, an on/off refrigeration control system has been implemented. This is done by turning on/off the compressor and the fan of the heat exchangers.

Table 6 reports the transducers specifications used (Coriolis effect mass flow rate meter, RTD 1004 wires thermo-resistances, piezoelectric absolute pressure gauge, wattmeter). The thermo-resistances are located outside the pipe, with a layer of heat transfer compound (aluminum oxide plus silicon) placed between the sensor and the pipe in order to provide good thermal contact. The whole pipe is covered with $25 \mathrm{~mm}$ thick flexible insulation. The system of temperature measurement was checked against a sensor positioned in pocket in a similarly insulated pipe work. For various test conditions, the difference between the two measurements has been always less than $0.3{ }^{\circ} \mathrm{C}$. The wattmeter is able to measure the electrical power absorbed by the compressor, the blowers and any kind of accessory installed for operation of the device. The energy consumption of the refrigeration system is measured by means of an energy meter. The test apparatus is equipped with 32 bit A/D converter acquisition cards linked to a personal computer that allows a high sampling rate $(10 \mathrm{kHz})$ 


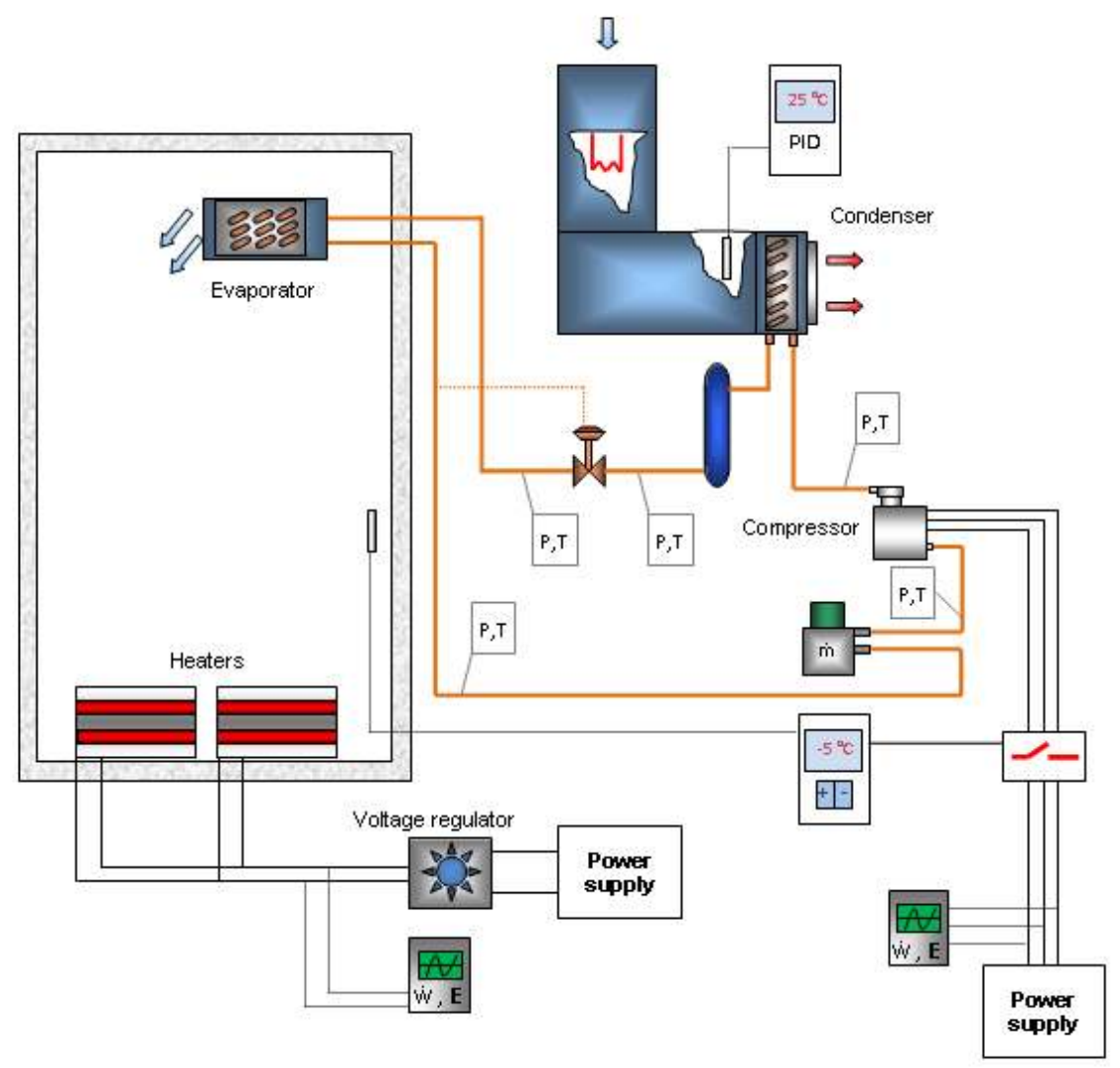

Figure 18. Sketch of the experimental plant

\begin{tabular}{|c|c|c|}
\hline Transducers & Range & Uncertainty \\
\hline Coriolis effect flowmeter & $0 \div 2 \mathrm{~kg} / \mathrm{min}$ & $+0.2 \%$ \\
\hline RTD 100 4 wires & $-100 \div 500{ }^{\circ} \mathrm{C}$ & $+0.15{ }^{\circ} \mathrm{C}$ \\
\hline Piezoelectric absolute pressure aguge & $1 \div 10 \mathrm{bar}$ & $+0.2 \%$ \\
& $1 \div 30 \mathrm{bar}$ & $+0.5 \% \mathrm{~F} . S$. \\
\hline Wattmeter & $0 \div 3 \mathrm{~kW}$ & $+0.2 \%$ \\
\hline Energy meter & $0 \div 1 \mathrm{MWh}$ & $+1 \%$ \\
\hline
\end{tabular}

Table 6. Transducers specifications 


\subsection{Experimental procedure}

We started the experimental investigation by analyzing the operation of the plant with R22. Subsequently, we retrofitted the refrigeration system with R422D in accordance with [31]. During the retrofitting operations, we changed the factory setting of the R22 thermostatic expansion valve in order to keep the operating superheat value for R422D in the same range used for R22, which was performed by turning the adjusting screw of the valve.

For both refrigerants, we used the same experimental procedure. Firstly, we proceeded with identifying the refrigerant charge necessary to guarantee that the fluid adequately wets the evaporator. For this purpose, we set the temperature of the air blown through the condenser to $24^{\circ} \mathrm{C}$ and the air inner to cold store to $-5^{\circ} \mathrm{C}$, while the cooling load was kept at $1000 \mathrm{~W}$. By means of a vacuum pump, we evacuated the circuit, and then we proceeded with introducing $0.40 \mathrm{~kg}$ of gas in the refrigerant circuit while the system was shut off to preserve the electrical motor of the compressor from overheating. Subsequently, we turned on the plant and the electrical heaters inside the cold store. During the operation of the plant, we monitored the value of the operating superheat, defined as the difference between the temperature at end of the evaporating process (considering the pressure drop into the evaporator) and temperature at the compressor inlet. Then, we systematically continued: additional $0.10 \mathrm{~kg}$ of refrigerant was introduced until, under steady state conditions, the operating superheat was not included in the range $7.0-10{ }^{\circ} \mathrm{C}$.

\begin{tabular}{|c|c|}
\hline Parameter & Value \\
\hline $\mathrm{H}$ & $950 \mathrm{~h} /$ year \\
\hline $\mathrm{PL}_{\mathrm{L}}$ & $10 \%$ \\
\hline $\mathrm{PR}_{\mathrm{R}}$ & 1 \\
\hline $\mathrm{V}$ & 1 year \\
\hline$\alpha$ & $0.6 \mathrm{~kg} \mathrm{CO} 2 / \mathrm{kWhe}$ \\
\hline $\mathrm{RC}_{\mathrm{R} 22}$ & $2.50 \mathrm{~kg}$ \\
\hline $\mathrm{R}_{\mathrm{R} 422 \mathrm{D}}$ & $2.30 \mathrm{~kg}$ \\
\hline
\end{tabular}

Table 7. Parameters in TEWI evaluation.

Once the system was charged to the specified value we proceeded with the evaluation of the energy consumption due to a year of operation (storage investigation). Usually, the service life $(\mathrm{V})$ of refrigeration system refers to the operation time of the equipment. If one is investigating the change of the environmental impact due to the retrofitting operations, the service life to use for the comparing analysis should not be equal to the actual life of the equipment, but one has to consider a reference service life. In this paper the reference service life is one year. In this scenario, the leakage rate per year has not to include the disposal percentage but only the accidental percentage due to operating conditions. Table 7 reports the parameters for TEWI evaluation according to eq.(1).

In particular, we investigated four different storage applications: $-5,0,5,10^{\circ} \mathrm{C}$. We considered as external air temperature reference the values reported in Table 8 , which represent typical conditions in Milan, Italy, used for our scenario. 
Since this table provides the daily change of external air temperature for each month, we planned 12 experiments each 24 hours long. The data reported in Table 8 were loaded as database for the PID controller, which modulates the voltage supply to the electrical resistances used to warm the air intake. For each experiment, recording of the energy consumption values started when the refrigeration control began to operate.

To evaluate the energetic performance, we needed further experiments aimed to analyze the behavior of the plant under steady state conditions (performance investigation). For this purpose, we planned 4 experiments at different storage temperatures: $-5,0,5,10^{\circ} \mathrm{C}$. During the experiments, we shunted the refrigeration control and we set the temperature of the air blown through the condenser to a reference value. We choose as reference the mean of the data reported in Table 8: $21{ }^{\circ} \mathrm{C}$. Usually, the start-up time required was about one hour. Steady state conditions were assumed to hold when the deviations of all controlled variables from their corresponding mean values were lower than $0.5^{\circ} \mathrm{C}$ for temperatures and $15 \mathrm{kPa}$ for pressures. At this stage, the test started and the logging of data with $0.5 \mathrm{~Hz}$ acquisition frequency was performed on all channels for $60 \mathrm{~s}$. For each channel, the 120 samples recorded were averaged. After $180 \mathrm{~s}$, each sample during $60 \mathrm{~s}$ was checked against the corresponding mean values of the two previous samples; when the mean values of the temperatures and of the pressures were within the range reported above, the steady state was reached.

January February March April May June July August September October November December

\begin{tabular}{|c|c|c|c|c|c|c|c|c|c|c|c|c|}
\hline Hours & {$\left[{ }^{\circ} \mathrm{C}\right]$} & {$\left[{ }^{\circ} \mathrm{C}\right]$} & {$\left[{ }^{\circ} \mathrm{C}\right]$} & {$\left[{ }^{\circ} \mathrm{C}\right]$} & {$\left[{ }^{\circ} \mathrm{C}\right]$} & {$\left[{ }^{\circ} \mathrm{C}\right]$} & {$\left[{ }^{\circ} \mathrm{C}\right]$} & {$\left[{ }^{\circ} \mathrm{C}\right]$} & {$\left[{ }^{\circ} \mathrm{C}\right]$} & {$\left[{ }^{\circ} \mathrm{C}\right]$} & {$\left[{ }^{\circ} \mathrm{C}\right]$} & {$\left[{ }^{\circ} \mathrm{C}\right]$} \\
\hline 0 & 11.4 & 12.7 & 15.7 & 17.5 & 19.9 & 21.6 & 22.2 & 22.35 & 21 & 18.6 & 15.1 & 12.2 \\
\hline 1 & 10.8 & 12.1 & 15.9 & 17.8 & 20.2 & 21.9 & 21.6 & 21.75 & 20.4 & 18 & 14.5 & 11.6 \\
\hline 2 & 10.2 & 11.5 & 14.5 & 16.3 & 18.7 & 20.4 & 21 & 21.15 & 19.8 & 17.4 & 13.9 & 11.0 \\
\hline 3 & 9.8 & 11.1 & 14 & 15.9 & 18.3 & 19.9 & 20.5 & 20.65 & 19.4 & 17 & 13.5 & 10.5 \\
\hline 4 & 9.4 & 10.7 & 13.7 & 15.5 & 17.9 & 19.6 & 20.1 & 20.25 & 19 & 16.6 & 13.1 & 10.1 \\
\hline 5 & 9.3 & 10.6 & 13.5 & 15.4 & 17.8 & 19.4 & 20 & 20.15 & 18.9 & 16.5 & 13 & 10.0 \\
\hline 6 & 9.5 & 10.8 & 13.8 & 15.6 & 18 & 19.7 & 20.2 & 20.35 & 19.1 & 16.7 & 13.2 & 10.3 \\
\hline 7 & 10.1 & 11.4 & 14.4 & 16.2 & 18.6 & 20.3 & 20.8 & 20.95 & 19.7 & 17.3 & 13.8 & 10.9 \\
\hline 8 & 11.2 & 12.5 & 15.5 & 17.3 & 19.7 & 21.4 & 21.9 & 22.05 & 20.8 & 18.4 & 14.9 & 11.9 \\
\hline 9 & 12.8 & 14.1 & 17 & 18.9 & 21.3 & 22.9 & 23.5 & 23.65 & 22.4 & 20 & 16.5 & 13.5 \\
\hline 10 & 14.6 & 15.9 & 18.8 & 20.7 & 23.1 & 24.7 & 25.3 & 25.45 & 24.2 & 21.8 & 18.3 & 15.3 \\
\hline 11 & 16.6 & 17.9 & 20.9 & 22.7 & 25.1 & 26.8 & 27.3 & 27.45 & 26.2 & 23.8 & 20.3 & 17.3 \\
\hline 12 & 18.5 & 19.8 & 22.8 & 24.6 & 27 & 28.7 & 29.2 & 29.35 & 28.1 & 25.7 & 22.2 & 19.3 \\
\hline 13 & 20.1 & 21.3 & 24.2 & 26.1 & 28.5 & 30.1 & 30.7 & 30.85 & 29.6 & 27.2 & 23.7 & 20.7 \\
\hline 14 & 20.9 & 22.2 & 25.2 & 27 & 29.4 & 31.1 & 31.6 & 31.75 & 30.5 & 28.1 & 24.6 & 21.7 \\
\hline 15 & 21.3 & 22.6 & 25.5 & 27.4 & 29.8 & 31.4 & 32 & 32.15 & 30.9 & 28.5 & 25 & 22.0 \\
\hline 16 & 20.9 & 22.2 & 25.2 & 27 & 29.4 & 31.1 & 31.6 & 31.75 & 30.5 & 28.1 & 24.6 & 21.7 \\
\hline 17 & 20.1 & 21.4 & 24.3 & 26.2 & 28.6 & 30.2 & 30.8 & 30.95 & 29.7 & 27.3 & 23.8 & 20.8 \\
\hline 18 & 18.8 & 20.1 & 23 & 24.9 & 27.3 & 28.9 & 29.5 & 29.65 & 28.4 & 26 & 22.5 & 19.5 \\
\hline 19 & 17.2 & 18.5 & 21.5 & 23.3 & 25.7 & 27.4 & 27.9 & 28.05 & 26.4 & 24.4 & 20.9 & 17.9 \\
\hline 20 & 15.6 & 16.9 & 19.9 & 21.7 & 24.1 & 25.8 & 26.4 & 26.55 & 25.2 & 22.8 & 19.3 & 16.4 \\
\hline 21 & 14.3 & 15.6 & 18.6 & 20.4 & 22.8 & 24.6 & 25 & 25.15 & 23.9 & 21.5 & 18 & 15.1 \\
\hline 22 & 13.1 & 14.4 & 17.4 & 19.2 & 21.6 & 23.3 & 23.8 & 23.95 & 22.7 & 20.3 & 16.8 & 13.9 \\
\hline 23 & 12.2 & 13.5 & 16.4 & 18.3 & 20.7 & 22.3 & 22.9 & 23.05 & 21.8 & 19.4 & 15.9 & 12.9 \\
\hline
\end{tabular}

Table 8. Change of the external air temperature for Milan (Italy). 


\subsection{Data elaboration}

To manage the large number of data, we developed a software application called FrigoCheck v.1.0. It is able to show in real time the coefficient of performance, the entropy and the enthalpy values of all points of the thermodynamic cycle. In addition, it shows the whole cycle on p-h diagram and it establishes the achievement of the steady state condition.

Since we measured the daily energy consumption for each month $(E d, i)$, we calculated the monthly energy consumption $(\mathrm{Em}, \mathrm{i})$ by means of the following equation

$$
\mathrm{E}_{\mathrm{m}, \mathrm{i}}=\mathrm{E}_{\mathrm{d}, \mathrm{i}} \times \mathrm{ND}_{\mathrm{i}}
$$

where the subscript $i$ refers to the generic month and ND is equal to the number of days for the i-th month. We obtained yearlong energy consumption (E) by summing the monthly energy consumptions $\left(\mathrm{Em}_{\mathrm{m}, \mathrm{i}}\right)$ :

$$
\frac{\dot{\mathrm{Q}}_{\mathrm{ref}}}{\mathrm{COP}}=\mathrm{E}=\sum_{\mathrm{i}} \mathrm{E}_{\mathrm{m}, \mathrm{i}}
$$

The uncertainty of the yearlong energy consumption is equal to $\pm 1 \%$.

Under steady state conditions, the overall efficiency performance of the plant is defined by means of the evaluation of COP, calculated as the ratio between the refrigeration capacity and the electrical power supplied to the plant (compressor, blowers and accessories):

$$
\mathrm{COP}=\frac{\dot{\mathrm{m}}\left(\mathrm{h}_{\mathrm{out}, \mathrm{EV}}-\mathrm{h}_{\mathrm{in}, \mathrm{EV}}\right)}{\dot{\mathrm{W}}_{\mathrm{el}}} .
$$

The COP accuracy has been equal to $\pm 2.5 \%$. In order to identify the efficiency of the plant, we considered the following ratio:

$$
\varepsilon=\frac{\text { COP }}{\frac{1}{\frac{\mathrm{T}_{\text {ex }}}{\mathrm{T}_{\text {cold }}}-1}} .
$$

\subsection{Scenario and sensitivity analysis for the TEWI difference}

Once completed the experimental investigation and the data elaboration, we focused our attention on the following question: after the retrofitting operations, how can one reduce the R422D TEWI? For this purpose, we considered a scenario and sensitivity analysis for the TEWI difference aimed to individuate the operating conditions (scenario) leading to the reduction of the TEWI. In equation (1), we individuated two parameters:

- Leakage rate per year, which acts on the direct effect;

- Energy saving, which acts on the indirect effect. 
For our first analysis, we kept the yearlong energy consumptions equal to those measured and varied the leakage rate per year, in accordance with the range $5-10 \%$. For each leakage rate value, we calculated the new R422D TEWI and compared it with the R22 TEWI by means of the following equation:

$$
\Delta \mathrm{TEWI}=\frac{\mathrm{TEWI}_{\mathrm{R} 422 \mathrm{D}}-\mathrm{TEWI}_{\mathrm{R} 22}}{\mathrm{TEWI}_{\mathrm{R} 22}} .
$$

We repeated this procedure for each test condition; therefore we obtained the change of the TEWI difference as a function of the leakage. As a second analysis, we kept the leakage rate equal to that reported in Table 7 and we varied the yearlong energy consumptions by considering an energy saving included in the range $0-100 \%$. Following a similar procedure to that above mentioned, we obtained the change of the TEWI difference at each test conditions as a function of the energy saving.

Since the one parameter analysis could lead to scenarios technically not feasible or very expensive, we deemed appropriate to consider the simultaneous change of both parameters.

\subsection{Results and discussions}

The first step of the experimental investigation led to identifying the correct charge for both refrigerants. As reported in Table 7, from experimental evaluation, the mass of R22 resulted $0.20 \mathrm{~kg}$ larger than that of R422D, which means an $8 \%$ reduction of refrigerant mass.

By means of the storage investigation we carried out the daily energy consumptions $\left(E_{d, i}\right)$ for each test conditions (Table 9).

\begin{tabular}{ccccccccc} 
& \multicolumn{9}{c}{ Daily energy consumption $\left(\mathrm{Ed}_{\mathrm{d}, \mathrm{i}}\right.$} & \multicolumn{2}{c}{$10^{\circ} \mathrm{C}$} \\
& $\mathrm{R} 22$ & $\mathrm{R} 422 \mathrm{D}$ & $\mathrm{R} 22$ & $\mathrm{R} 422 \mathrm{C}$ & $\mathrm{R} 22$ & $\mathrm{R} 422 \mathrm{C}$ & $\mathrm{R} 22$ & $\mathrm{R} 422 \mathrm{D}$ \\
& $(\mathrm{Wh})$ & $(\mathrm{Wh})$ & $(\mathrm{Wh})$ & $(\mathrm{Wh})$ & $(\mathrm{Wh})$ & $(\mathrm{Wh})$ & $(\mathrm{Wh})$ & $(\mathrm{Wh})$ \\
January & $4^{\prime} 848$ & $5^{\prime} 080$ & $3^{\prime} 051$ & $3^{\prime} 392$ & $1^{\prime} 800$ & $2^{\prime} 097$ & $1^{\prime} 039$ & $1^{\prime} 334$ \\
February & $5^{\prime} 017$ & $5^{\prime} 280$ & $3^{\prime} 166$ & $3^{\prime} 529$ & $1^{\prime} 873$ & $2^{\prime} 188$ & $1^{\prime} 086$ & $1^{\prime} 395$ \\
March & $5^{\prime} 430$ & $5^{\prime} 772$ & $3^{\prime} 448$ & $3^{\prime} 868$ & $2^{\prime} 053$ & $2^{\prime} 416$ & $1^{\prime} 202$ & $1^{\prime} 547$ \\
April & $5^{\prime} 701$ & $6^{\prime} 098$ & $3^{\prime} 635$ & $4^{\prime} 093$ & $2^{\prime} 173$ & $2^{\prime} 567$ & $1^{\prime} 279$ & $1^{\prime} 648$ \\
May & $6^{\prime} 075$ & $6^{\prime} 551$ & $3^{\prime} 893$ & $4^{\prime} 405$ & $2^{\prime} 339$ & $2^{\prime} 779$ & $1^{\prime} 387$ & $1^{\prime} 791$ \\
June & $6^{\prime} 348$ & $6^{\prime} 884$ & $4^{\prime} 082$ & $4^{\prime} 636$ & $2^{\prime} 461$ & $2^{\prime} 937$ & $1^{\prime} 468$ & $1^{\prime} 897$ \\
July & $6^{\prime} 436$ & $6^{\prime} 991$ & $4^{\prime} 143$ & $4^{\prime} 710$ & $2^{\prime} 500$ & $2^{\prime} 987$ & $1^{\prime} 494$ & $1^{\prime} 931$ \\
August & $6^{\prime} 461$ & $7^{\prime} 022$ & $4^{\prime} 161$ & $4^{\prime} 731$ & $2^{\prime} 512$ & $3^{\prime} 002$ & $1^{\prime} 501$ & $1^{\prime} 941$ \\
September & $6^{\prime} 246$ & $6^{\prime} 759$ & $4^{\prime} 011$ & $4^{\prime} 549$ & $2^{\prime} 415$ & $2^{\prime} 878$ & $1^{\prime} 437$ & $1^{\prime} 857$ \\
October & $5^{\prime} 864$ & $6^{\prime} 295$ & $3^{\prime} 747$ & $4^{\prime} 229$ & $2^{\prime} 245$ & $2^{\prime} 660$ & $1^{\prime} 326$ & $1^{\prime} 710$ \\
November & $5^{\prime} 346$ & $5^{\prime} 671$ & $3^{\prime} 390$ & $3^{\prime} 798$ & $2^{\prime} 016$ & $2^{\prime} 369$ & $1^{\prime} 178$ & $1^{\prime} 516$ \\
December & $4^{\prime} 944$ & $5^{\prime} 193$ & $3^{\prime} 116$ & $3^{\prime} 470$ & $1^{\prime} 842$ & $2^{\prime} 149$ & $1^{\prime} 066$ & $1^{\prime} 369$
\end{tabular}

Table 9. Daily energy consumption measured. 
Considering equation (2) we converted the results shown in Table 9 in monthly energy consumptions $\left(\mathrm{Em}_{\mathrm{m}, \mathrm{i}}\right)$ and then in yearlong energy consumptions (Fig. 19).

It can be seen that the yearlong energy consumption pertaining to R422D is larger than that of R22 (7.10-28.9\%) for each test conditions. Furthermore, for both refrigerants the energy consumption diminishes with the increase of the air temperature inner cold store. This is easily understandable if one considers the reduction of the operating temperature span, which is defined as the mean difference between the hot thermal sink (external ambient) and the cold thermal sink (cold store).

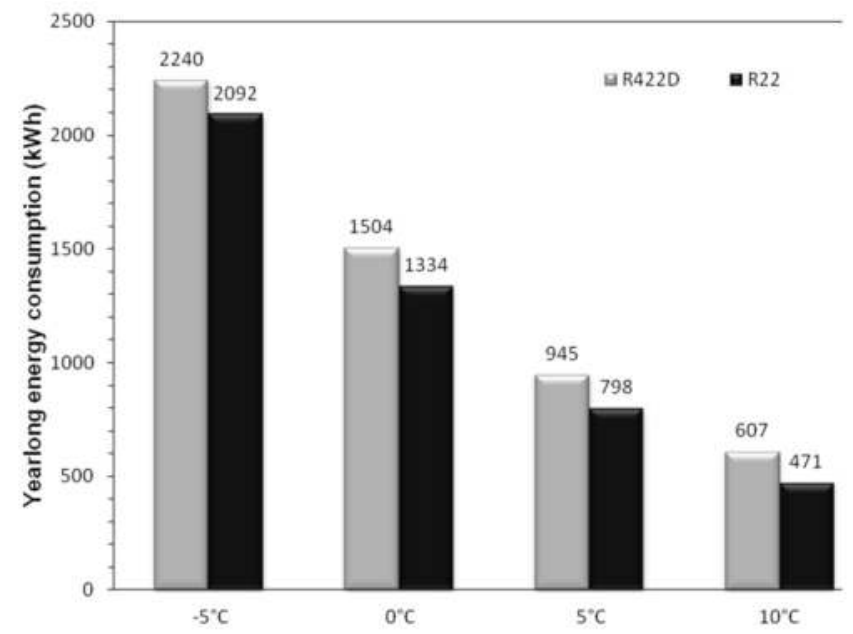

Figure 19. Yearlong energy consumption vs. air temperature inner to cold store $( \pm 1 \%)$.

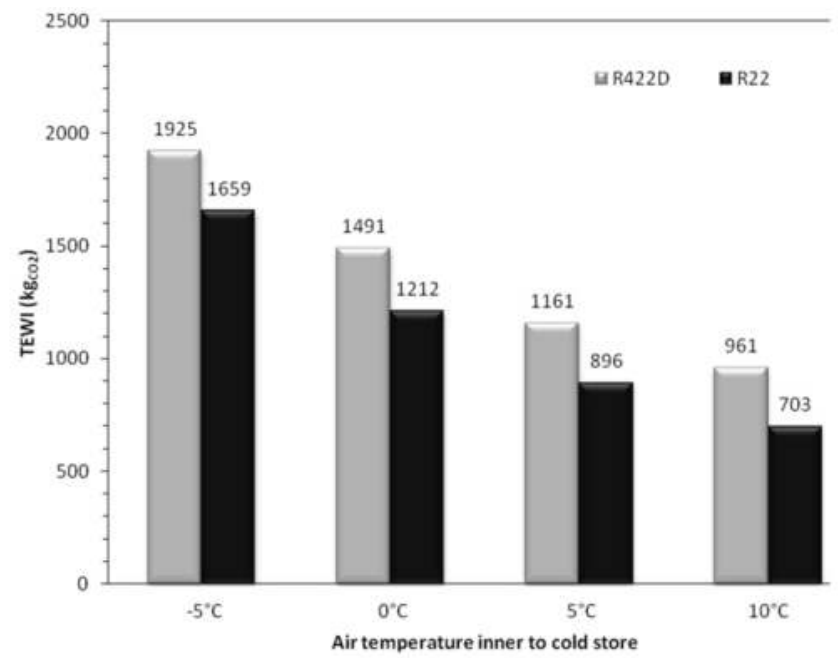

Figure 20. TEWI vs. air temperature inner to cold store $( \pm 10 \%)$. 
Monthly energy consumption $\left(\mathrm{E}_{\mathrm{m}, \mathrm{i}}\right)$

\begin{tabular}{ccccccccc} 
& \multicolumn{2}{c}{$-5{ }^{\circ} \mathrm{C}$} & \multicolumn{2}{c}{$0^{\circ} \mathrm{C}$} & \multicolumn{2}{c}{$+5^{\circ} \mathrm{C}$} & \multicolumn{2}{c}{$10^{\circ} \mathrm{C}$} \\
& $\mathrm{R} 22$ & $\mathrm{R} 422 \mathrm{D}$ & $\mathrm{R} 22$ & $\mathrm{R} 422 \mathrm{D}$ & $\mathrm{R} 22$ & $\mathrm{R} 422 \mathrm{D}$ & $\mathrm{R} 22$ & $\mathrm{R} 422 \mathrm{D}$ \\
& $(\mathrm{Wh})$ & $(\mathrm{Wh})$ & $(\mathrm{Wh})$ & $(\mathrm{Wh})$ & $(\mathrm{Wh})$ & $(\mathrm{Wh})$ & $(\mathrm{Wh})$ & $(\mathrm{Wh})$ \\
January & $150^{\prime} 295$ & $157^{\prime} 473$ & $94^{\prime} 568$ & $105^{\prime} 144$ & $55^{\prime} 809$ & $64^{\prime} 994$ & $32^{\prime} 220$ & $41^{\prime} 354$ \\
February & $140^{\prime} 481$ & $147^{\prime} 833$ & $88^{\prime} 637$ & $98^{\prime} 814$ & $52^{\prime} 451$ & $61^{\prime} 272$ & $30^{\prime} 406$ & $39^{\prime} 060$ \\
March & $168^{\prime} 338$ & $178^{\prime} 940$ & $106^{\prime} 893$ & $119^{\prime} 904$ & $63^{\prime} 650$ & $74^{\prime} 888$ & $37^{\prime} 250$ & $47^{\prime} 951$ \\
April & $171^{\prime} 040$ & $182^{\prime} 945$ & $109^{\prime} 037$ & $122^{\prime} 777$ & $65^{\prime} 177$ & $77^{\prime} 024$ & $38^{\prime} 368$ & $49^{\prime} 452$ \\
May & $188^{\prime} 323$ & $203^{\prime} 066$ & $120^{\prime} 670$ & $136^{\prime} 553$ & $72^{\prime} 494$ & $86^{\prime} 164$ & $43^{\prime} 002$ & $55^{\prime} 517$ \\
June & $190^{\prime} 455$ & $206^{\prime} 521$ & $122^{\prime} 471$ & $139^{\prime} 068$ & $73^{\prime} 833$ & $88^{\prime} 105$ & $44^{\prime} 029$ & $56^{\prime} 907$ \\
July & $199^{\prime} 505$ & $216^{\prime} 713$ & $128^{\prime} 433$ & $145^{\prime} 995$ & $77^{\prime} 511$ & $92^{\prime} 610$ & $46^{\prime} 299$ & $59^{\prime} 862$ \\
Agust & $200^{\prime} 298$ & $217^{\prime} 685$ & $128^{\prime} 984$ & $146^{\prime} 668$ & $77^{\prime} 868$ & $93^{\prime} 070$ & $46^{\prime} 534$ & $60^{\prime} 173$ \\
September & $187^{\prime} 370$ & $202^{\prime} 755$ & $120^{\prime} 329$ & $136^{\prime} 463$ & $72^{\prime} 449$ & $86^{\prime} 327$ & $43^{\prime} 119$ & $55^{\prime} 708$ \\
October & $181^{\prime} 794$ & $195^{\prime} 150$ & $116^{\prime} 157$ & $131^{\prime} 084$ & $69^{\prime} 589$ & $82^{\prime} 448$ & $41^{\prime} 105$ & $53^{\prime} 019$ \\
November & $160^{\prime} 377$ & $170^{\prime} 141$ & $101^{\prime} 711$ & $113^{\prime} 953$ & $60^{\prime} 490$ & $71^{\prime} 070$ & $35^{\prime} 335$ & $45^{\prime} 467$ \\
December & $153^{\prime} 268$ & $160^{\prime} 987$ & $96^{\prime} 591$ & $107^{\prime} 557$ & $57^{\prime} 091$ & $66^{\prime} 604$ & $33^{\prime} 037$ & $42^{\prime} 425$
\end{tabular}

Table 10. Monthly energy consumption calculated by means of the Eq. 2

In Fig. 20, for both refrigerants we have drawn the TEWI relative to one year of operation as function of the air temperature inner to cold store. Since the first term of the equation (1) is constant for both refrigerants and for each test condition, it is noticeable that the change of TEWI is directly influenced by that of the energy consumption.

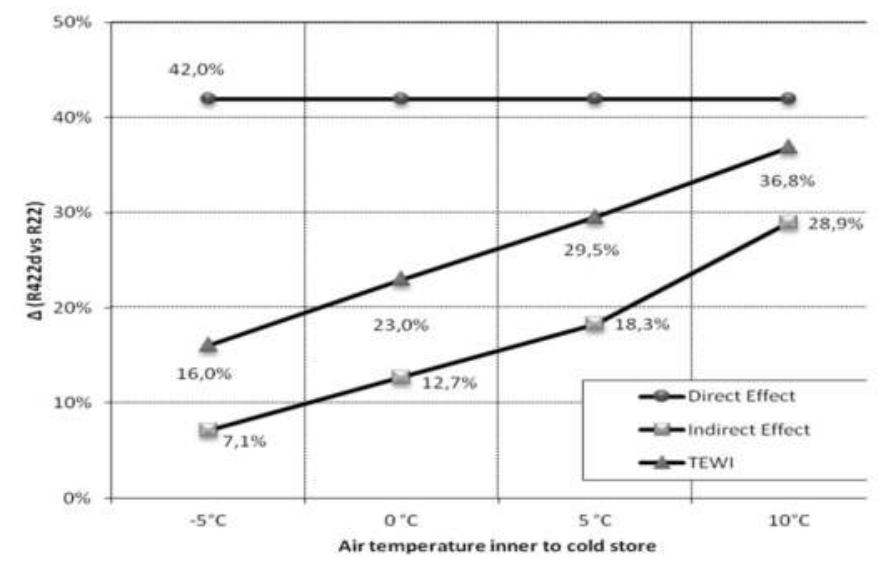

Figure 21. Difference percentage of indirect, direct and TEWI vs. air temperature inner to cold store.

As remarked in Fig.21, the adoption of R422D leads to a worsening of the environmental impact. Because the GWP of R422D is $50 \%$ higher than that of R22, the $8 \%$ reduction of refrigerant mass does not impact significantly the decrease of the direct effect of R422D, which results equal to $42 \%$ higher than that of R22. The indirect effect (Fig. 21) of R422D is higher than that of R22; in particular, this difference grows with the increase of the storage 
temperature. Consequently, the release of $\mathrm{CO}_{2}$, due to the adoption of $\mathrm{R} 422 \mathrm{D}$, grows from a minimum of $7.1 \%$ to a maximum of $28.9 \%$.

The augmentation of the energy consumption has been also confirmed by performance investigation. In Fig. 22, we report both the change of the COP and that of the efficiency as a function of the air temperature inner to cold store. The results there illustrated highlights a lower efficiency of the plant due to the adoption of R422D. It is possible to observe that COP increases with the increase of the air temperature inner to cold store and that the COP for R22 is higher than for R422D.

In particular, the difference between the COP for R22 and that for R422D is, on average, 20\% and it increases with the increase of the air temperature inner cold store. The worsening of the energy performance, due to the use of R422D as substitute of R22, is remarked by the efficiency values showed in Fig. 22.

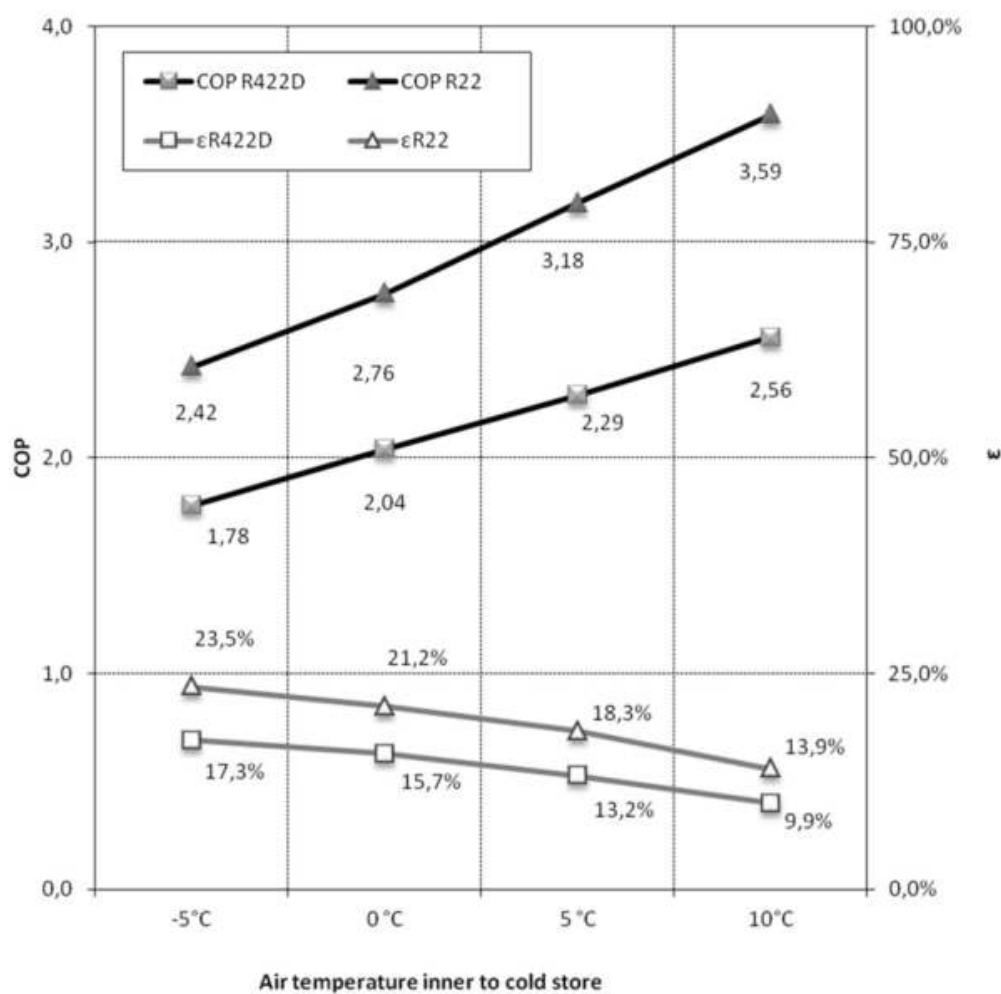

Figure 22. COP and efficiency of the plant vs. air temperature inner to cold store $( \pm 2.5 \%)$.

The lower R422D efficiency values represent a warning on the inefficiency of the plant subsequent to the retrofitting operations. During the performance investigation, we observed a substantially different behavior of the condenser. As reported in Fig. 23 the condensing pressure of R422D was higher than that of R22, while the evaporating pressure 
for both refrigerants was similar. This gain in terms of pressure at condenser shows that, when R422D is used as refrigerant, the heat exchange surface of the condenser is insufficient to reject the thermal power. Furthermore, a more high condensing pressure leads to an increase of electrical power absorbed by the compressor further reducing the COP. However, it is our interest to understand how to improve the energy performance of the plant operating with R422D, with the intent of reducing the indirect impact on the environment. Particular attention should be given to the condenser, since, as highlighted above, the heat exchange surface required by $\mathrm{R} 22$ is less than that required by R422. Referring to the operation with R422D, an improvement of the heat exchange at condenser could lead to reduce the condensing pressure, and then the specific work of compressor. Furthermore, the fluid leaving the condenser would be sub-cooled further, allowing a gain of the specific heat of evaporation. Consequently, a lower mass flow rate could be required, and then the power absorbed by the compressor could be reduced, guarantying an improvement of the COP.

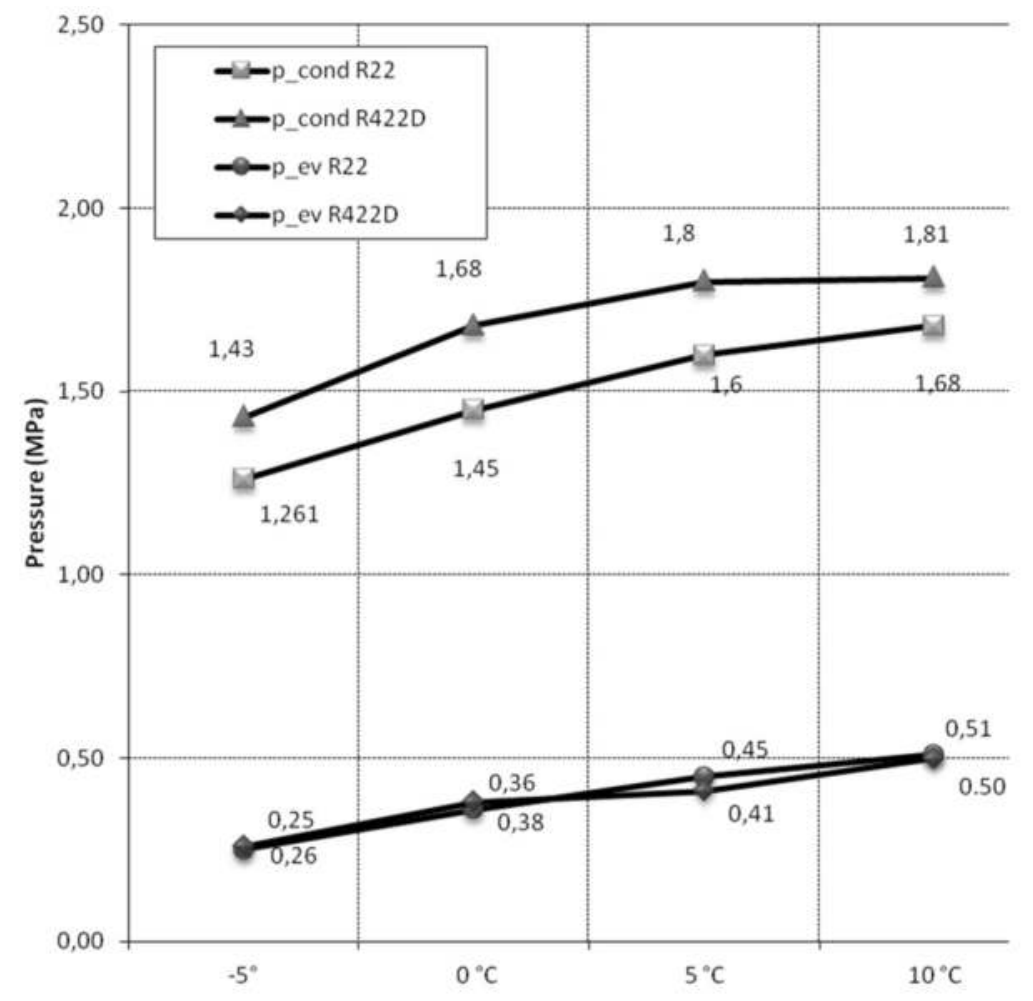

Air temperature inner to cold store

Figure 23. Condensing and evaporating pressure vs. air temperature inner to cold store. 
To enhance the heat exchange at condenser two methods could be considered:

- if the blower have different operating speeds, select the highest speed available, otherwise, change the blower with another having higher volumetric capacity;

- $\quad$ replace the condenser with one having a larger surface.

It is important to underline that the first solution is better than second, since cheaper and technically easier, but it could lead to a larger absorption of electrical power by blowers, negatively affecting the COP. Based on theoretical considerations [32], it is possible to expect a $15 \%$ increase of COP for a 1.5 bar reduction of the condensing pressure.

When R422D is used as refrigerant, a further improvement of the energy performance could be obtained by installing an electronic expansion valve instead of the thermostatic one. As showed by Lazzarin and Noro [33], for any refrigerants the electronic expansion valve allows a lower condensation pressure in systems equipped with air-cooled condensers, thanks to the ability of monitoring the variations of theoutside air temperature. Consequently, they [33] indicated, on average, an $8 \%$ reducing of energy consumption for Mediterranean locations and a 15\% for NorthEuropean locations.

Once completed the experimental investigation, we considered a new scenario still to evaluated a sensitivity analysis for the TEWI difference, now aimed to highlight the way to reduce the environmental impact of the R22 retrofit with R422D. For this purpose, we have reported in Fig. 24 the change of the TEWI difference percentage as a function of the leakage rate per year, while in Fig. 25 we have done it as a function of the energy saving per year. For both figures, we have identified three scenarios:

- Scenario A: it represents the parameter domain, for which for every test conditions the environmental impact for R422D becomes higher than that for R22.

- Scenario B: it represents the parameter domain of transition, for which at least for one test condition the environmental impact for R422D becomes lower than that for R22.

- $\quad$ Scenario C (or eco-friendly scenario): it represents the parameter domain, for which for every test conditions the environmental impact for R422D becomes lower than that for R22.

Furthermore, we reported two dashed axes, whose intersection represents the breakeven point between the TEWI of R22 and that of R422D for all test conditions. Fig. 24 shows that for leakage rate per year lower than 5.7\% the TEWI of R422D becomes lower than that of R22. The scenario B is very narrow and this allows achieving of the breakeven point almost simultaneously for all operating conditions. Considering $5 \%$ as technical limit for nohermetical plant, it can see that the scenario C (Fig. 24) occurs for leakage rate per year values including in the narrowest range $5.0 \div 5.4 \%$, and it leads to a maximum $\triangle T E W I$ (absolute value) included between $-6.0 \div-2.0 \%$. The scenario $\mathrm{C}$ is technically feasible but it leads to an increase of the management costs: leakage check more frequent could lead to reduce the leakage rate, as indicated in [34]. 
Considering the change of the energy saving (Fig. 25), it can be seen that the scenario B is very large and it starts for $\sim 20 \%$ energy saving; the breakeven point is reachable for $\sim 70 \%$ energy saving. These results are not reassuring, because a $70 \%$ energy saving should correspond to a plant efficiency equal to $30 \%$, when usually, for actual plant operating under the same conditions here investigated, the efficiency can be equal to $25 \%$. Differently, as above mentioned, $20 \%$ energy saving could be obtained as a result both of the heat exchange improvement and of the use of electronic expansion valves. Since both the strong reduction of leakage rate and the high energy saving lead to an increase of the total cost of the retrofitting operations, it is necessary to consider an overlay parameter change. For this purpose, we have developed another scenario considering the simultaneous change of both parameters.

In Fig. 26 we have reported four different charts, each referring to different test condition in terms of air temperature inner to cold store. For each chart, we have drawn a solid black line, which identifies the border between the scenario characterized by a negative TEWI difference (eco-friendly scenario) and that by a positive TEWI difference. This time the ecofriendly scenario is identified by means of a $2 \mathrm{D}$ domain (leakage rate \& energy saving): one has to select one couple of values for leakage rate and energy saving per year in order to obtain a reduction of TEWI consequently to the adoption of R422D. That the overlaying of the effects allows obtaining strong TEWI reduction by means of cheap operations: minor reductions of leakage rate and energy saving are required.

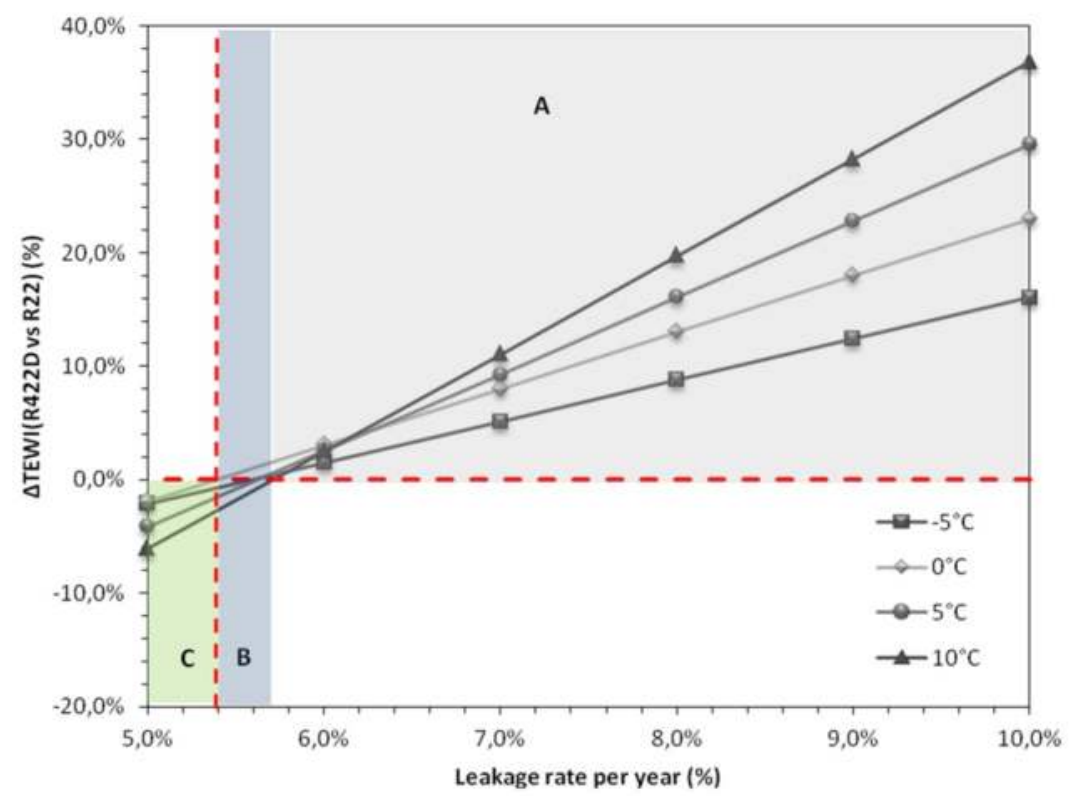

Figure 24. Difference of TEWI vs. leakage rate per year and operating scenarios. 


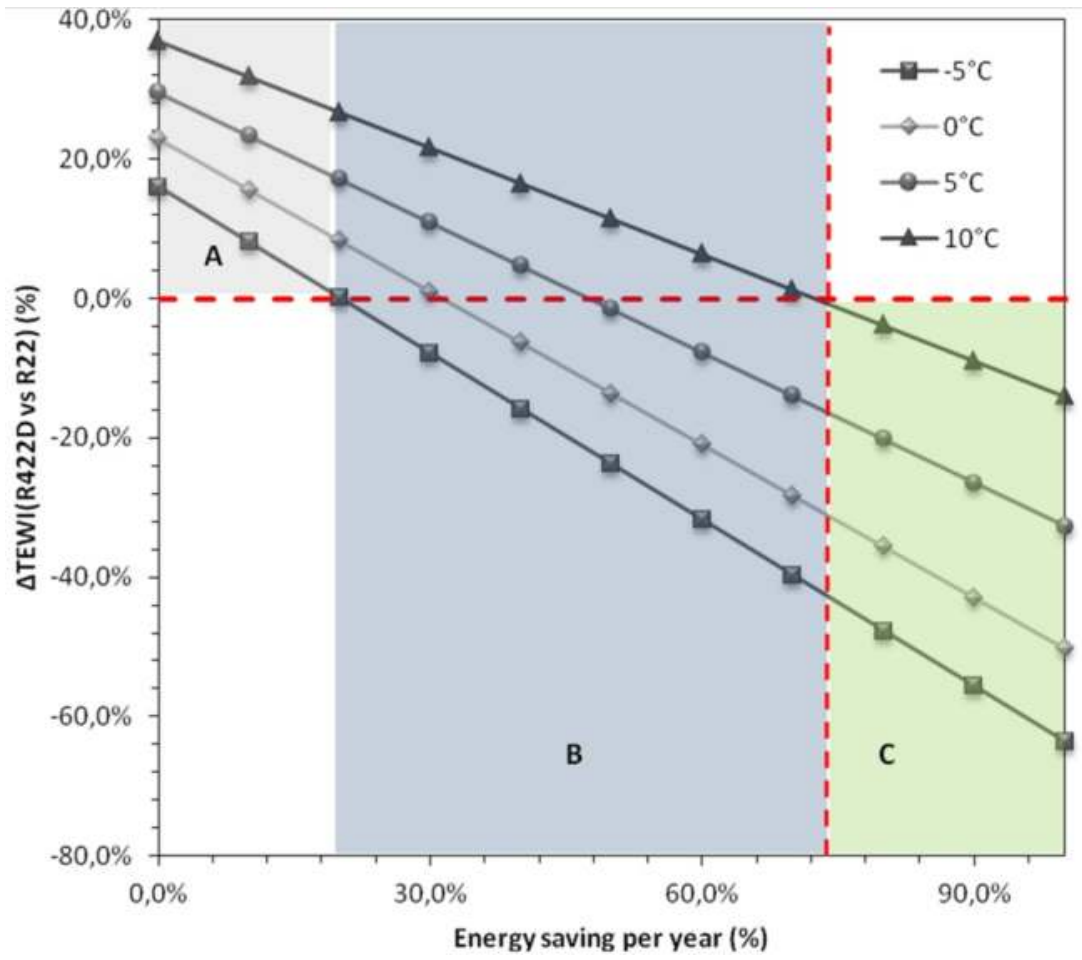

Figure 25. Difference of TEWI vs. energy saving per year and operating scenarios.

\subsection{Concluding remarks}

An experimental investigation has been carried out study the environmental impact of the R22 retrofit with R422D and to draw possible eco-friendly scenarios [35]. The experimental investigation consisted of two parts:

- $\quad$ Storage investigation, aimed at developing of TEWI analysis.

- Performance investigation, aimed at analyzing the behavior of the plant under steady state conditions.

For both investigations, we have considered four operating conditions in terms of the air temperature inner to cold store: $-5,0,5,10^{\circ} \mathrm{C}$. To emulate actual operating conditions we choose Milan as reference locality. 
a)
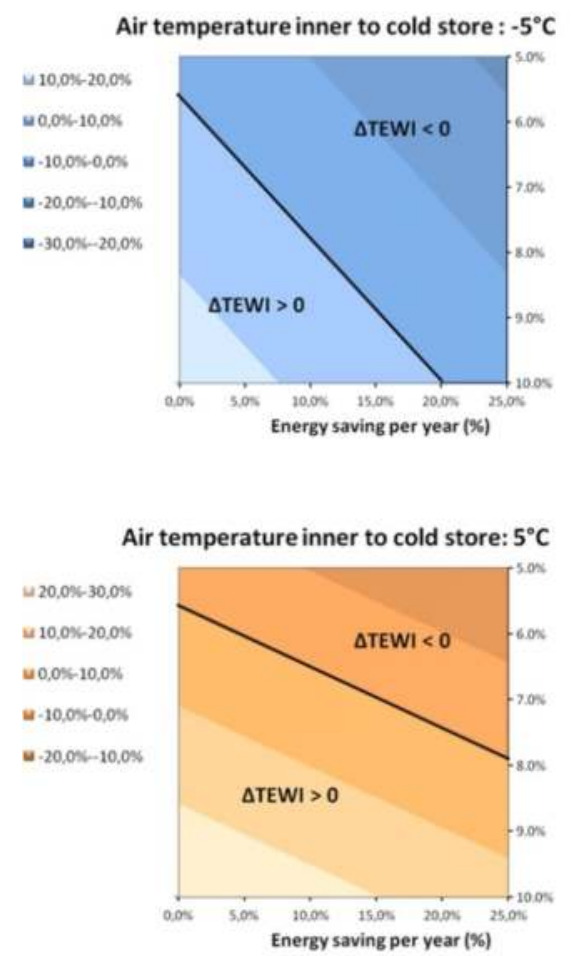

c) b)
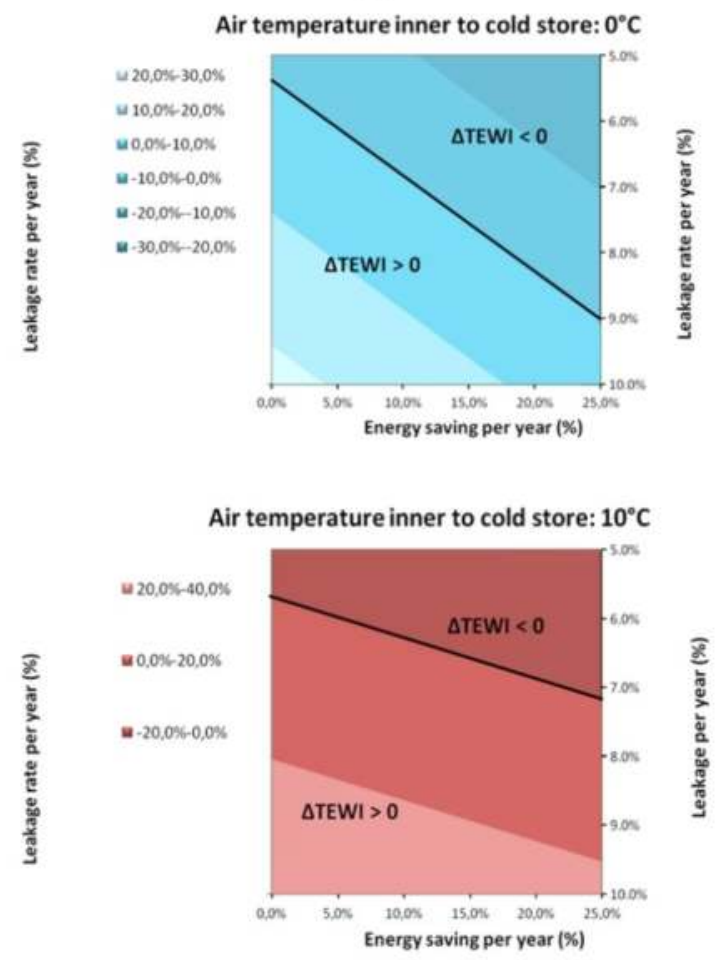

d)

Figure 26. Operating scenario charts a) at $-5^{\circ} \mathrm{C}$, b) at $0{ }^{\circ} \mathrm{C}$, c) $5^{\circ} \mathrm{C}$ and d) $10^{\circ} \mathrm{C}$.

Subsequently, a scenario and sensitivity analysis for the TEWI difference has been introduced to study ways of reducing the environmental impact of the R22 retrofit with R422D. For this purpose leakage rate and improved efficiency have been considered as parameters and three parametric analyses have been developed: two have been carried out by changing only one parameter per time, while the other one by changing simultaneously both parameters. Based on our investigation, we can draw the following conclusions:

1. The storage investigation have demonstrated that for each test conditions the R22 retrofit with $\mathrm{R} 422 \mathrm{D}$ leads to an increase of the energy consumption up to $28.9 \%$ worsening $\mathrm{CO}_{2}$ emissions. 
2. Since the GWP of R422D is much higher than that of R22 and even if the charge of $\mathrm{R} 422 \mathrm{D}$ is $8 \%$ lower than that of R22, the direct effect of the R422D is $42 \%$ higher than that of R22.

3. As a consequence of the R22 retrofit with R422D, the plant investigated has shown an increase of TEWI up to $36.8 \%$.

4. The performance investigation highlighted that the operation with R422D is less efficient than that with R22. In particular, the difference between the COP for R22 and that for R422D is, on average, $20 \%$, and it grows with the raising of the air temperature of the inner cold store.

5. R22 retrofit with R422D leads to an increase of the condensing pressure, which indicates that the heat exchange surface of the condenser is insufficient to reject the thermal power, worsening the efficiency.

6. To improve the energy performance and then to reduce the indirect effect, we proposed two ways: improving of the heat exchange surface and adoption of electronic expansion valves. Based on theoretical considerations it is possible to obtain a $20 \%$ reduction of energy consumption.

7. The scenario and sensitivity analysis for the TEWI difference have demonstrated that for each test condition there are some operating eco-friendly scenarios. In particular, if the parameters change simultaneously, the eco-friendly scenario results technically feasible: both lower reductions of leakage rate and lower energy saving are required.

\section{An experimental evaluation of the greenhouse effect in R134a substitution with R744}

\subsection{R134a substitution with R744}

In the field of the mobile refrigeration systems, the European Parliament already set a regulation of F-Gases phase out that bans the use of refrigerants having GWPs exceeding 150. Such regulation is in effect since 2011.

R134a is an HFC with zero ODP and a GWP of 1300. According to the above mentioned European regulation on F-Gases, the use of R134a will be banned in mobile systems. R744 is a natural fluid $\left(\mathrm{CO}_{2}\right)$ therefore with no ODP and negligible direct contribution to global warming and can be a substitute of R134a. In this study, the impact of the substitution of R134a with R744 on global warming was studied through experimental evaluations of the TEWI index under different operating conditions.

The experimental tests discussed in this study compare a commercial R134a refrigeration plant subjected to a cold store and a prototype R744 system working as a classical spitsystem to cool air in a trans-critical cycle.

Table 11 reports the parameters adopted for the TEWI evaluation. The annual operating hours in the TEWI simulation are 8760. These correspond to a commercial refrigerator cold store according to Dir.94/2/CE [36]. 


\begin{tabular}{|c|c|}
\hline Parameter & Value \\
\hline $\mathrm{H}$ & $8760 \mathrm{~h}$ \\
\hline $\mathrm{PL}$ & $5 \%$ year \\
\hline $\mathrm{PR}$ & $95 \%$ \\
\hline $\mathrm{V}$ & 10 years \\
\hline$\alpha$ & $0.6 \mathrm{~kg} \mathrm{CO} 2 / \mathrm{kWh}_{\mathrm{e}}$ \\
\hline
\end{tabular}

Table 11. Parameters for TEWI evaluation.

\subsection{Experimental equipment}

\subsubsection{Refrigeration plant working with carbon dioxide}

Figure 27 shows a sketch of the experimental plant.

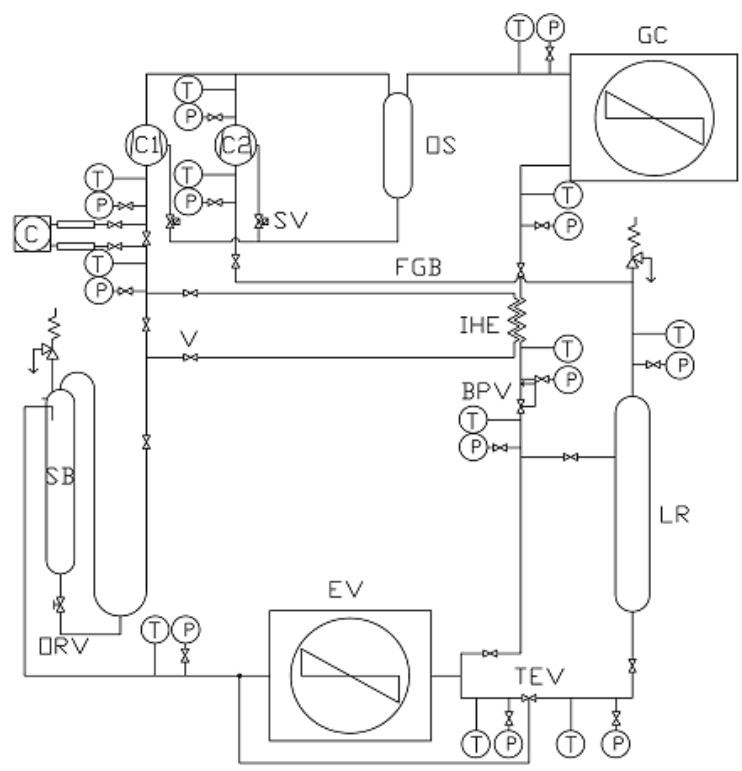

\begin{tabular}{|c|c|c|}
\hline \multirow{4}{*}{$\begin{array}{l}< \\
\leftarrow \\
\leftarrow\end{array}$} & \multicolumn{2}{|r|}{ Legend } \\
\hline & c & Coriolis mass flow rate gauge \\
\hline & $\mathrm{C} 1$ & Compressor \\
\hline & $\mathrm{ce}$ & Auxiliary Compressor \\
\hline \multirow{14}{*}{$\begin{array}{l}\text { electrical } \\
\text { resistances }\end{array}$} & $\mathrm{BPV}$ & Back-Pressure Valve \\
\hline & FGB & Flash Gas Bypass \\
\hline & GC & Gas Cooler \\
\hline & EV & Evaporator \\
\hline & IHE & Internal Heat Exchanger \\
\hline & LR & Liquid Receiver \\
\hline & QRL & Dil Regolator Valve \\
\hline & QS & Dil Separator \\
\hline & $P$ & Pressure sensor \\
\hline & sV & Solenaid Valve \\
\hline & SB & Suction Buffer \\
\hline & & Temperature sensor \\
\hline & TEV & Thermostatic Expansion Valve \\
\hline & $v$ & Valve \\
\hline
\end{tabular}

Figure 27. Sketch of the carbon dioxide experimental plant. 
Basically, there are two single-stage hermetic reciprocating compressors, an oil separator, an air gas-cooler, a liquid capacity, an air evaporator, an electronic expansion valve (EEV) and an electronically-regulated back pressure valve (BPV). The main compressor is a semihermetic one. At evaporation temperatures of $5^{\circ} \mathrm{C}$ and of $30^{\circ} \mathrm{C}$ at the gas-cooler exit, when the pressure is 80 bar, the refrigerating power is about $3000 \mathrm{~W}$. An internal heat exchanger (IHX) between the refrigerant flow at the compressor suction and at the exit of the gascooler is provided. The lamination occurs thanks to the back-pressure valve and to the electronic expansion one. An auxiliary circuit can be used to by-pass the back-pressure valve, in order to vary the evaporation temperature. The air temperature on the condenser is regulated by an air-flow driven by a blower in a thermally insulated channel. Its temperature is modulated by some electrical resistances. This simulates variable external conditions, as well.

The plant is fully instrumented, in order to evaluate its performance as a whole, as well as that of each single component. The pressure and the temperature of the carbon dioxide are measured both at the inlet and at the outlet of each device. Mass flow rate is monitored at the main compressor suction (see Figure 27). Two watt transducers are used to measure the electrical power supplied to the compressors. Table 12 summarizes all the characteristics of the plant instrumentation.

\begin{tabular}{|lll|}
\hline Transducers & Range & Uncertainty \\
\hline Coriolis effect flowmeter & $0 \div 2 \mathrm{~kg} / \mathrm{min}$ & $\pm 0.2 \%$ \\
Piezoelectric absolute pressure & $1 \div 100 \mathrm{bar}$ & $\pm 0.4 \%$ \\
gauge & & \\
RTD 1004 wires & $-100 \div 500{ }^{\circ} \mathrm{C}$ & $\pm 0.15^{\circ} \mathrm{C}$ \\
Wattmeter & $0.5 \div 6 \mathrm{~kW}$ & $\pm 0.2 \%$ \\
\hline
\end{tabular}

Table 12. Transducers specifications.

The Coefficient of Performance is evaluated with eq. (4), with uncertainty of $\pm 3.8 \%$ according to the procedure suggested by Moffat [37].

The uncertainty of the TEWI was calculated by applying the error propagation theory to Eq. (1). A balance is used to measure the refrigerant charge with an uncertainty of $\pm 0.2 \%$ in the range $0-100 \mathrm{~kg}$. Sand et al. [18] suggested that a minimum of $20 \%$ uncertainty exists for the GWP values assigned to refrigerants by the Intergovernmental Panel on Climate Change (IPCC). These uncertainties, when combined with other estimates and assumptions of the analysis lead to a TEWI uncertainty of $10 \%$.

\subsubsection{Refrigeration plant working with $R 134 a$}

The experimental vapour compression plant, subjected to a commercially available cold store and reported in Figure 28, is made up of a semi-hermetic reciprocating compressor. 


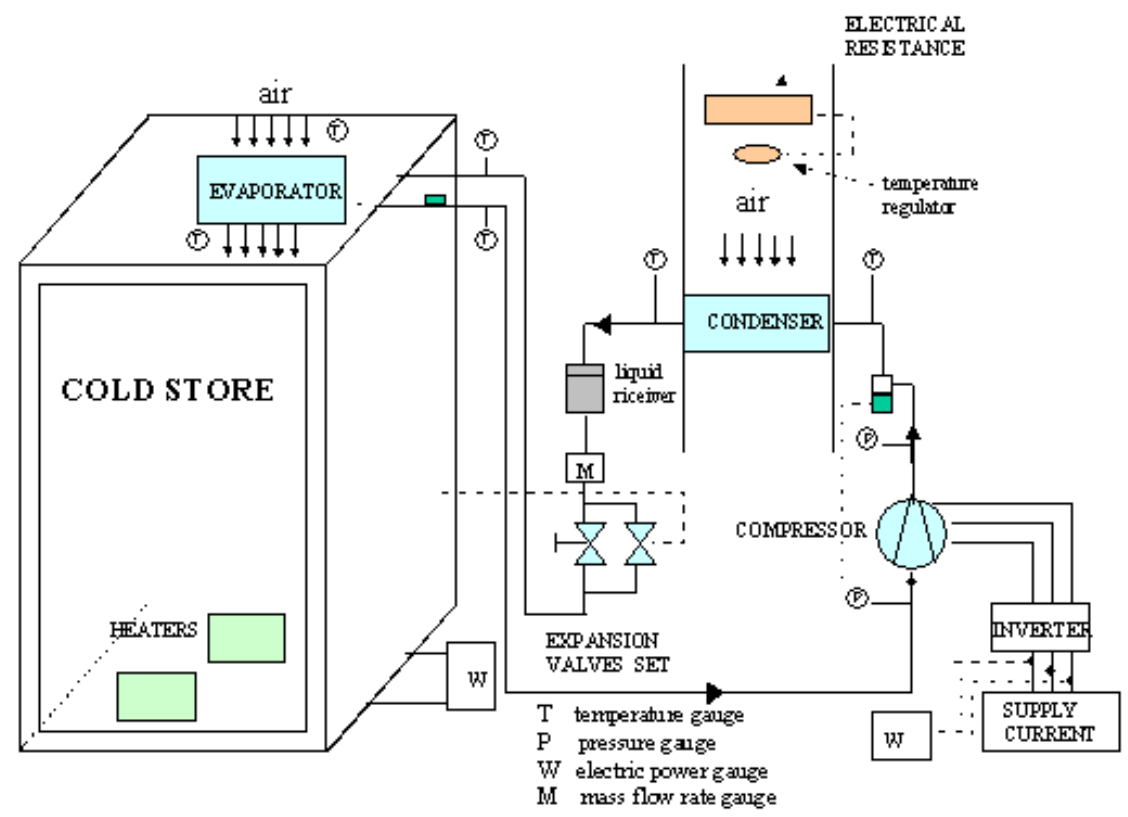

Figure 28. Sketch of the R134a experimental plant.

It was designed for the fluid R134a, according to the manufacturer specifications. The plant is supplied with a three-phase current ( $380 \mathrm{~V}$ phase-phase), an air condenser followed by a liquid receiver, a manifold with two expansion valves, a thermostatic one and a manual one mounted in parallel, to feed an air cooling evaporator inside the cold store.

In the evaporation temperature range $-20 \div 10^{\circ} \mathrm{C}$ with a $35^{\circ} \mathrm{C}$ condensing temperature, working with the R134a at the nominal frequency of $50 \mathrm{~Hz}$, the compressor refrigerating capacity varies in the range $1.4 \div 4.4 \mathrm{~kW}$. A blower provides an air-flow within a thermally insulated channel where some electrical resistances are located. Their power-modulation enables the control of the air temperature on the condenser and simulates different external conditions.

The refrigeration duty in the cold store is simulated by means of regulated electrical resistances. The electric power is measured by means of a watt transducer whose specifications are reported in Table 13.

Further specifications of the experimental plant were reported in a previous work [38-41]. The COP values calculated in accordance with (7), should be considered with an uncertainty less than $\pm 0.5 \%$. The smaller accuracy in the calculus of the COP pertaining to the R744 plant is due to a different accuracy of the pressure gauges used for the R744, as compared to those used for the R134. The lower accuracy of the pressure gauges for the R744 stems from the larger measurement range required. 


\begin{tabular}{|ccc|}
\hline Transducers & Range & Uncertainty \\
\hline Coriolis effect flowmeter & $0 \div 2 \mathrm{~kg} / \mathrm{min}$ & $\pm 0.2 \%$ \\
RTD 100 4 wires & $-100 \div 500{ }^{\circ} \mathrm{C}$ & $\pm 0.15^{\circ} \mathrm{C}$ \\
Piezoelectric absolute pressure & $1 \div 10 \mathrm{bar}$ & $\pm 0.2 \%$ \\
gauge & $1 \div 30 \mathrm{bar}$ & $\pm 0.5 \% \mathrm{~F} . \mathrm{S}$ \\
Wattmeter & $0 \div 3 \mathrm{~kW}$ & $\pm 0.2 \%$ \\
\hline
\end{tabular}

Table 13. Transducers specifications.

\subsection{Results and discussion}

The experimental, R744 plant was optimized in order to maximize its energetic performance.

The direct contribution to global warming was evaluated on the basis of the measured plant charge. The R744 charge is the optimal one, corresponding to $6.87 \mathrm{~kg}$. That for R134a is of $2.45 \mathrm{~kg}$. The direct contribution to the greenhouse effect pertaining to R134a during the plant useful life is always greater that that pertaining to R744. The direct contribution of R744 is negligible respect to the indirect one, whereas that of R134a is comparable.

System performances are compared for two evaporation temperatures of 0 and $5{ }^{\circ} \mathrm{C}$, respectively, by varying the temperature of the external air over the gas-cooler and the condenser. Both plants develop a refrigerant power of $3000 \mathrm{~W}$.

In the evaluation of the total contribution to greenhouse effect, three scenarios were considered: a commercial refrigerator cold store, a classical split-system and a mobile refrigeration system.

In the first scenario a commercial refrigeration cold store was considered. According to Dir.94/2/CE, it works for 8760 annual operating hours.

Figure 29 reports TEWI as a function of external air temperature for an evaporating temperature of 0 and $5{ }^{\circ} \mathrm{C}$, respectively. Figure clearly shows that, for both refrigerant fluids, TEWI increases with the external air temperature because of the COP decrease.

Indeed, COP decreases due to the decrease of the refrigerant fluid enthalpy at the evaporator inlet because of the pressure increase at the condenser/gas-cooler. Its rise leads to a higher enthalpy at the compressor outlet, thus increasing the specific work of compression. The COP decrease for R744 is more marked than that of R134a.

The performance of both refrigerant fluids at fixed external air temperature decreases with evaporating temperature. This effect is more marked for the plant working with the transcritical cycle. In a typical transcritical cycle the expansion valve provides a dominant contribution to the overall energy loss $[42,43]$. The irreversibility of the expansion process increases by decreasing the evaporating pressure 
Figure 29 clearly shows that, for a refrigerating system working as a commercial refrigerator cold store, the TEWI pertaining to R134a is always lower than that of R744 from a minimum of $-22 \%$ to a maximum of $-73 \%$. The higher indirect contribution to global warming of R744 with respect to R134a always prevails.

The second scenario is a classical split-system, that according to Dir.29/1/2003 works with 500 annual operating hours.

Figure 30 reports TEWI as a function of external air temperature for an evaporating temperature of 0 and $5{ }^{\circ} \mathrm{C}$.

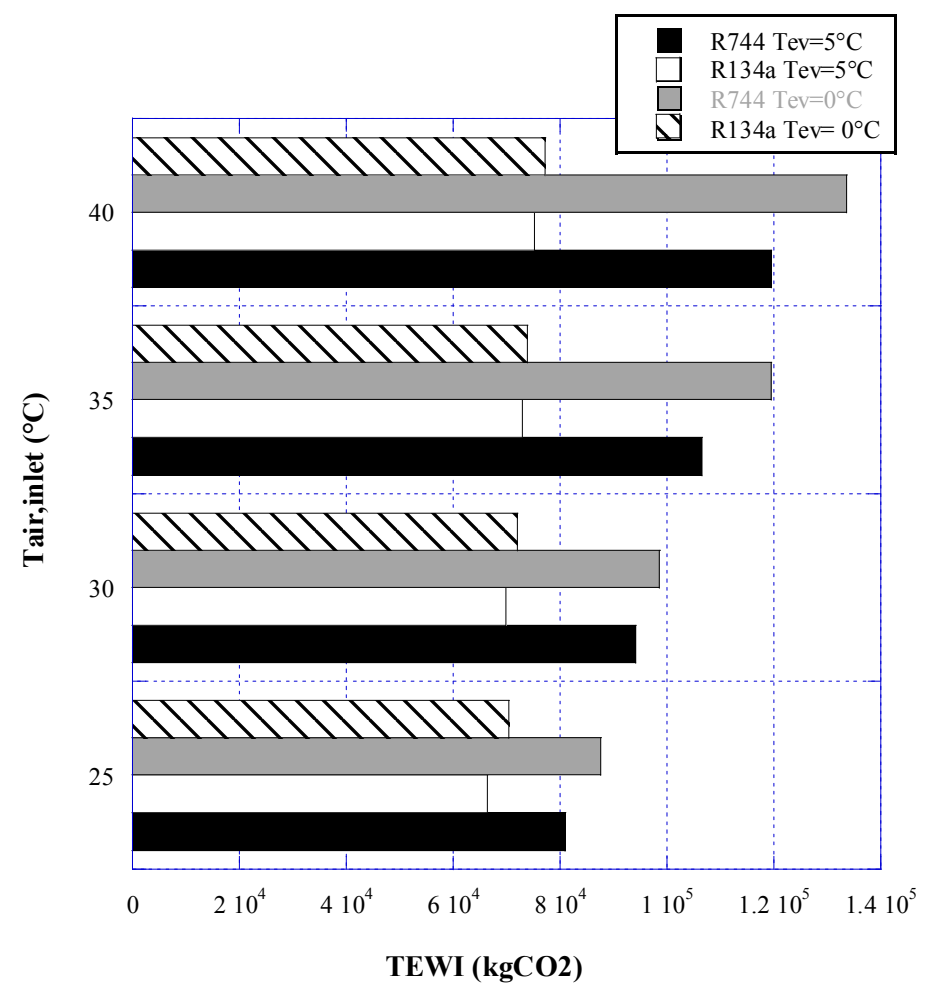

Figure 29. TEWI as a function of air temperature at the inlet of the condenser/gas-cooler, for two evaporating temperature, in a commercial refrigerator cold store. 


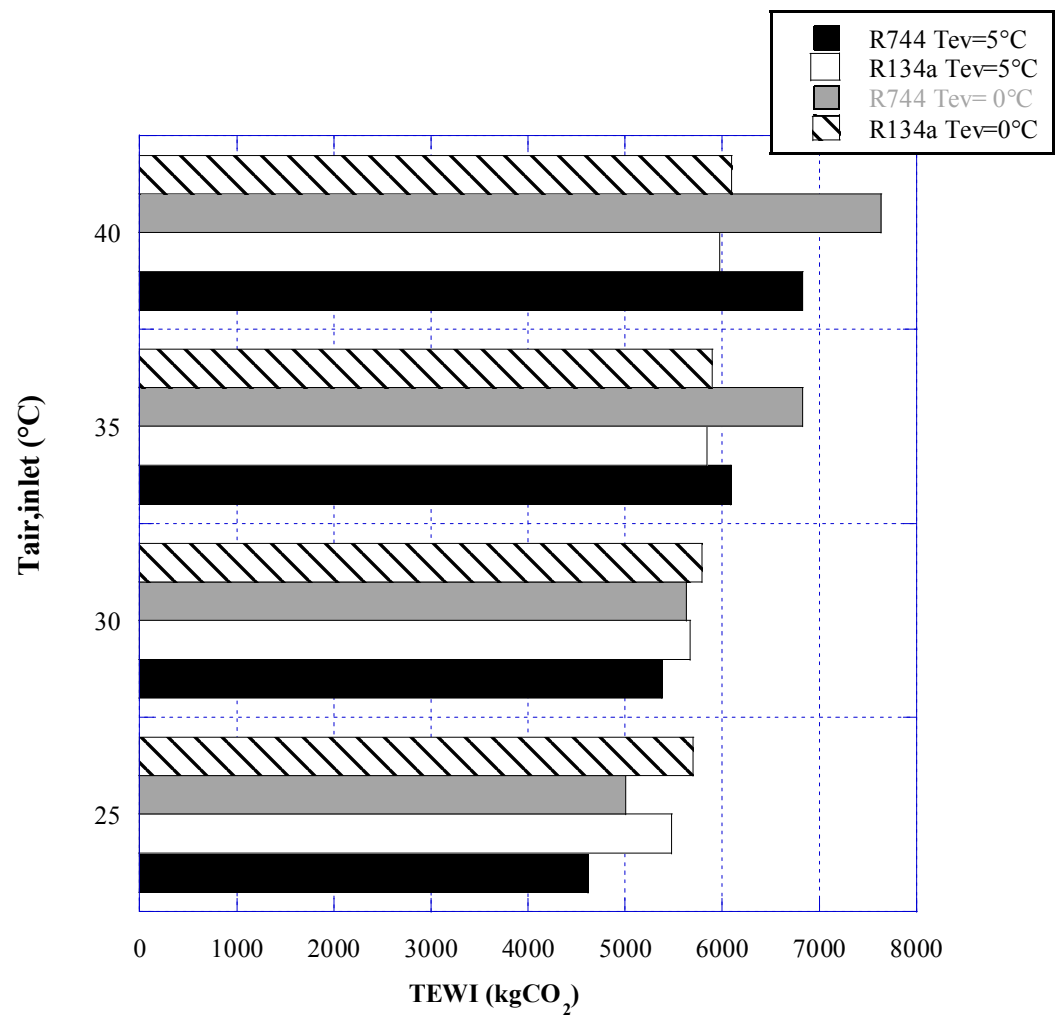

Figure 30. TEWI as a function of air temperature at the inlet of the condenser/gas-cooler, for two evaporating temperature, in a classical split-system.

In this case TEWI of R134a is higher than that of R744 for external air temperature of 25 and $30{ }^{\circ} \mathrm{C}$ (from a minimum of 3 to maximum of $16 \%$ ). In these experimental conditions the system working with R744 has a lower global warming impact as compared to a system with R134a. This effect is due to the lower direct contribution of R744 that prevails on the indirect one. In the operating conditions corresponding to external air temperature of 35 and $40{ }^{\circ} \mathrm{C}$, the TEWI of R134a is lower that that of R744 (from a minimum of -4 to maximum of $-25 \%)$.

The third scenario is a mobile refrigeration system

In the following analysis, a $1000 \mathrm{~kg}$ small commercial car was considered. For this car, $98 \mathrm{~g}$ of $\mathrm{CO}_{2}$ are released per $\mathrm{km}[46]$ in a typical urban cycle with a mean engine power of $5 \mathrm{~kW}$. On this basis, the fraction of $\mathrm{CO}_{2}$ emission produced by a $3 \mathrm{~kW}$ air conditioning device employing R134a was evaluated. The R134a charge is $800 \mathrm{~g}$. That of $\mathrm{CO}_{2}$ is $1.20 \mathrm{~kg}$.

From these data and from the previous experimental results, it is possible to evaluate the TEWI for both R134a and R744. 
In figure $31 \mathrm{TEWI}$ is reported as a function of the refrigerant leakage rate per year.

The figure clearly shows that TEWI of R134a is lower than that of R744 (from a minimum of -4.7 to a maximum of $-22 \%$ ) in the yearly refrigerant leakage rate of 5 and $20 \%$, respectively. At $25 \%$ the two fluids show the same TEWI. On the contrary, at 30\% leakage, the R134a TEWI exceeds that of R744 of $+4 \%$.

In a typical mobile air conditioning device, the yearly refrigerant leakage rate is around $25 \%$.

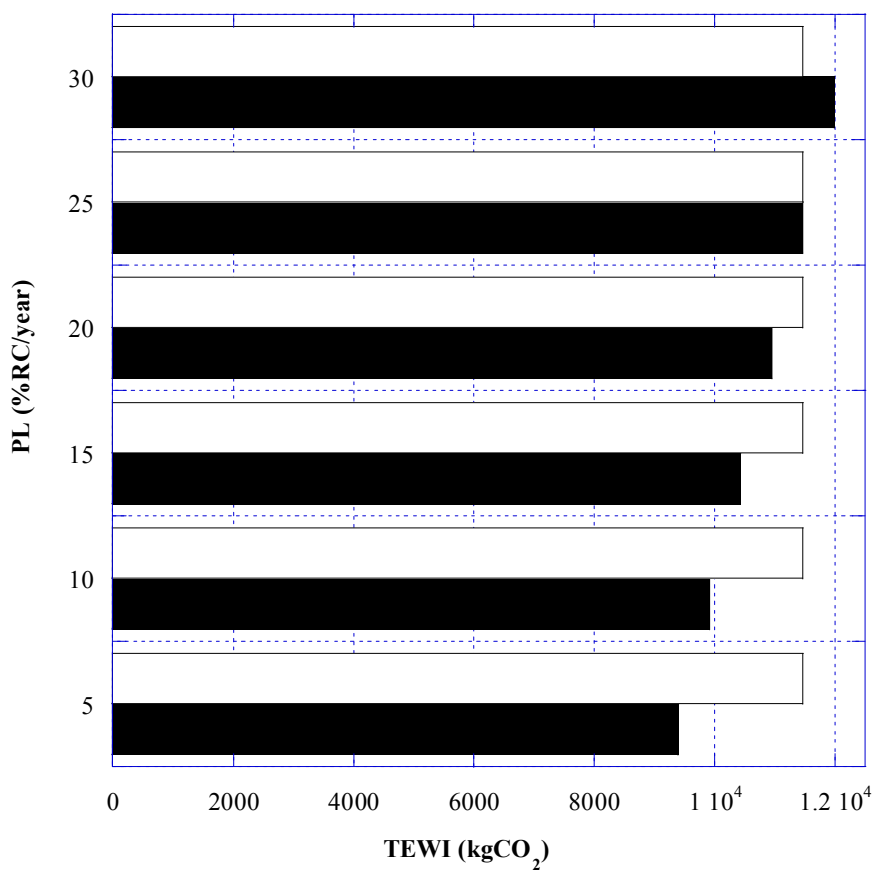

Figure 31. TEWI as a function of refrigerant leakage rate in a mobile refrigeration system.

\subsection{Concluding remarks}

The substitution of R134a was analyzed in terms of global warming effect. Indeed, R134a will be banned in mobile systems, according to the regulation on F-Gases. R134a has a relevant direct global warming effect stemming from its absorption power of long-wave radiations, that depends on its GWP and on the fraction of refrigerant charge released into 
the atmosphere. A possible substitute of R134a could be carbon dioxide, i.e. a natural fluid with negligible direct contribution to global warming.

An experimental comparison between R134a and R744 was performed in terms of the total equivalent warming impact (TEWI) that combines the effect of the direct emissions of refrigerants with the indirect effect of energy consumption.

The experiments compare a commercial R134a refrigeration plant subjected to a cold store and a prototype R744 system working in a trans-critical cycle. A preliminary analysis was carried out, in order to maximize the energetic performance of the trans-critical cycle.

In the comparison, different, common working conditions were considered by varying the external temperature and that of the evaporation. In all the test runs, the energetic efficiency of the trans-critical cycle is always lower than that of the plant working with R134a. This leads to a greater indirect global warming effect of the plant working with R744.

Three different scenarios were considered: a commercial refrigerator cold store, a classical split-system and a mobile refrigeration system.

Based on our investigation, the following conclusions can be drawn:

1) For a refrigerating system working as a commercial refrigerator cold store at fixed refrigerant leakage rate per year (5\%), the TEWI pertaining to R134a is always lower than that of R744 by a minimum of $-22 \%$ up to a maximum of $-73 \%$.

2) A refrigerating system working as a classical split (at fixed 5\% refrigerant leakage rate per year) with R134a has a greater global warming impact as compared to a system with R134a for external air temperature of 25 and $30{ }^{\circ} \mathrm{C}$ (from a minimum of 3 to maximum of $16 \%$ ). Whereas, in the operating conditions corresponding to external air temperature of 35 and $40{ }^{\circ} \mathrm{C}$, the TEWI of R134a is lower that that of R744 (from a minimum of -4 to maximum of $-25 \%)$.

3) In a mobile refrigeration system, the TEWI of R134a is lower than that of R744 (from a minimum of -4.7 to a maximum of $-22 \%$ ) for yearly refrigerant leakage rates between 5 and $20 \%$. At $25 \%$ leakage, the two fluids show the same TEWI, whereas, at $30 \%$ leakage, the R134a TEWI exceeds that of R744 by $+4 \%$. In view of these results, R744 does not appear to be a suitable substitute for R134a. The latter, however, must be ruled out in mobile systems, in any case. Therefore, alternative fluids such as HFO (HydroFluoro-Olefin) should be considered.

\section{Conclusions and recommendations}

The impact of vapour compression refrigerant fluids on global warming has been analyzed by means of an experimental study. Vapour compression plants have both a direct and an indirect contribution to global warming. The Total Equivalent Warming Impact (TEWI) index takes into account both the direct and indirect contribution to global warming. 
In this chapter the substitution of R22 and R134a has been studied. R407C and R422D are possible substitutes for R22. R22 is an HCFC, with an ODP of 0.05 and a GWP of 1700 . Both refrigerant fluids are drop in substitutes: R407C is an HFC with zero ODP and a GWP 6\% lower than that of R22, R422D is an HFC with zero ODP and a GWP 31\% greater than that of R22. R134a is a single hydrofluorocarbon (HFC) compound. It has no chlorine content and therefore no ozone depletion potential, and a GWPR134a of 1300.

In the field of mobile refrigeration systems, the European Parliament (EU 842/2006) already set F-Gases phase out regulation that bans the use of refrigerants having GWPs in excess of 150. According to this regulation the use of R134a will be banned in mobile systems. Pure $\mathrm{CO}_{2}$ (R744), with no direct contribution to global warming, can be a substitute of R134a. Experimental tests have been carried out on different vapour compression pilot plants for a range of operating conditions, the prototype R744 system works in a trans-critical cycle

From the experimental analysis the following general conclusions can be drawn:

- R22 direct contribution to the greenhouse effect is greater than that pertaining to R407C $(+15 \%)$. The COP corresponding to R407C is 3.3-19\% lower than that pertaining to R22.

- In the experimental tests corresponding to a condensation temperature in the range 43 / $47^{\circ} \mathrm{C}$ and to an evaporation temperature in the range $-12 /-7^{\circ} \mathrm{C}$, the TEWI pertaining to $\mathrm{R} 407 \mathrm{C}$ exceeds that of R22 by about $11 \%$. Therefore, the substitution of R22 with R407C should be unacceptable if specific reference is made to the greenhouse effect.

- In the experimental tests corresponding to a condensation temperature in the range $53 / 58^{\circ} \mathrm{C}$ and to an evaporation temperature inlet range $2 / 10^{\circ} \mathrm{C}, \mathrm{R} 22$ and $\mathrm{R} 407 \mathrm{C}$ behave in a similar way as regards the greenhouse effect.

- For high evaporation and condensation (over $50^{\circ} \mathrm{C}$ ) temperatures, the TEWI of R407C is slightly lower than that of R22. As a consequence, the substitution of R22 with R407C is favourable, since no harm is produced to the ozone layer and no increase in $\mathrm{CO}_{2}$ emission is made.

- GWP of R422D is much higher than that of R22 and even if the charge of R422D is $8 \%$ lower than that of R22, the direct effect of the R422D is 42\% higher than that of R22.

- The plant working with R422D is less efficient than that with R22. In particular, the difference between the COP for R22 and that for R422D is, on average, $20 \%$, and it grows with the raising of the air temperature of the inner cold store.

- For each test conditions the R22 retrofit with R422D leads to an increase of the energy consumption up to $28.9 \%$, worsening $\mathrm{CO}_{2}$ emissions, with an increase of TEWI up to $36.8 \%$. Therefore the substitution of R22 with R422D is always unacceptable from the point of view of greenhouse effect.

- In all the test runs, the energetic efficiency of the trans-critical cycle is always lower than that of the plant working with R134a. This leads to a greater indirect global warming effect of the plant working with R744.

- For a refrigerating system working as a commercial refrigerator cold store the substitution of R134a with R744 is always unacceptable because the TEWI pertaining to $\mathrm{R} 134 \mathrm{a}$ is always lower than that of R744 (from $-22 \%$ to $-73 \%$ ). 
- A refrigerating system working as a classical split with R134a has a greater global warming impact as compared to a system with R744 for external air temperature of 25 and $30{ }^{\circ} \mathrm{C}$ (from a minimum of 3 to maximum of $16 \%$ ). Whereas, in the operating conditions corresponding to external air temperature of 35 and $40{ }^{\circ} \mathrm{C}$, the TEWI of $\mathrm{R} 134 \mathrm{a}$ is lower that that of R744 (from a minimum of -4 to maximum of $-25 \%$ ).

- In a mobile refrigeration system, the TEWI of R134a is lower than that of R744 (from a minimum of -4.7 to a maximum of $-22 \%$ ) for yearly refrigerant leakage rates between 5 and $20 \%$. At $25 \%$ leakage, the two fluids show the same TEWI, whereas, at $30 \%$ leakage, the R134a TEWI exceeds that of R744 by $+4 \%$. In view of these results, R744 does not appear to be a suitable substitute for R134a.

\section{Nomenclature}

\section{Symbols}

$\mathrm{CO}_{2, \mathrm{dir}}=$ direct contribution to global warming $(\mathrm{kgCO} 2)$

$\mathrm{CO}_{2 \text {, indir }}$ indirect contribution to global warming $(\mathrm{kgCO})$

$\mathrm{COP}=$ Coefficient Of Performance

$\mathrm{E}=$ energy consumption $(\mathrm{kWh})$

GWP = Global Warming Potential ( $\left.\mathrm{kgCO}_{\mathrm{Cg}} \mathrm{kgfrigerant}\right)$

$\mathrm{H}=$ annual operating hours (h/years)

$\mathrm{h}=$ enthalpy $(\mathrm{kJ} / \mathrm{kg})$

$\dot{m}=$ refrigerant mass flow rate $(\mathrm{kg} / \mathrm{s})$

ODP $=$ Ozone Depletion Potential

$\mathrm{p}=$ pressure $(\mathrm{Pa})$

$\mathrm{P}_{\mathrm{L}}=$ accidental refrigerant leaks per year (\% refrigerant charge/year)

$\mathrm{P}_{\mathrm{R}}=$ recycling rate $(\%$ refrigerant charge $)$

$\mathrm{Q}_{\mathrm{ref}}=$ refrigerant power $(\mathrm{kW})$

$\mathrm{RC}=$ refrigerant charge $(\mathrm{kg})$

$\mathrm{T}=$ temperature $\left({ }^{\circ} \mathrm{C}, \mathrm{K}\right)$

TEWI $=$ Total Equivalent Warming Impact $(\mathrm{kgCO})$

$\mathrm{V}=$ plant useful life (years)

$\dot{W}=$ compression power $(\mathrm{kW})$

\section{Greek symbols}

$\alpha=\mathrm{CO}_{2}$ emission from power conversion ( $\left.\mathrm{kgCO}_{2} / \mathrm{kWhe}\right)$

$\beta=$ compression ratio

$\triangle \mathrm{TEWI}=\mathrm{TEWI}$ difference

$\varepsilon=$ efficiency

\section{Subscripts}

air $=$ air 
$\mathrm{amb}=$ external ambient

$\mathrm{CO}=$ condenser

cold $=$ air inner to cold store

$\mathrm{D}=$ daily

el= electrical

$\mathrm{EV}=$ evaporator

hot $=$ external air ambient

$\mathrm{i}=\mathrm{i}$-th

in= inlet

$\mathrm{m}=$ monthly

MT = mean thermodynamic

out $=$ outlet

ref $=$ refrigerant

$\mathrm{w}=$ water

$\mathrm{wg}=$ water glycol mixture

\section{Author details}

C. Aprea* and A. Maiorino

Dipartimento di Ingegneria Industriale, Università di Salerno, via Ponte Don Melillo, Fisciano, Salerno, Italia

A. Greco

DETEC, Università degli Studi di Napoli Federico II, P.le Tecchio, Napoli, Italia

\section{References}

[1] M.J. Molina, F.S. Rowland, 1974, Stratospheric sink for chlorofluoromethanes: Chlorine atom catalyzed destruction of ozone, Nature n. 249, pp.810-812.

[2] C. Muller, 1993, Atmospheric ozone and greenhouse gases observation: an update, Proc. Int. Conf. Energy Efficiency Refrig. and Global Warming Impact, Commission B1/2 IIR, University of Ghent, Belgium, pp. 45-54.

[3] Montreal Protocol on Substances That Deplete the Ozone Layer, 1987. United Nations (UN), New York, NY, USA (1987 with subsequent amendments).

[4] UNEP, 2007a. Decisions Adopted by the Nineteenth Meeting of the Parties to the Montreal Protocol on Substances that Deplete the Ozone Layer. United Nations Environment Programme (UNEP) Ozone Secretariat, Nairobi, Kenya, (2007)

[5] S. Fisher, F. A. Creswick, 1988, Energy use impact of chlorofluorocarbon alternatives, ORNL/CON-273.

[6] S. Fisher, F. A. Creswick, 1988, How will CFC bans affect energy use, ASHRAE Journal, vol. 30, n. 11.

${ }^{*}$ Corresponding Author 
[7] Brohan, P., Kennedy, J.J., Harris, I., Tett, S.F.B., Jones, P.D., Uncertainty estimates in regional and global observed temperature changes: a new dataset from 1850, J. of Geophysical Res. 111, (2006), D12106.

[8] Rayner, N.A., Brohan, P., Parker, D.E., Folland, C.K., Kennedy, J.J., Vanicek, M., Ansell, T.J., Tett, S.F.B, Improved analyses of changes and uncertainties in marine temperature measured in situ since the mid-nineteenth century: the HadSST2 dataset. Journal of Climate 19, (2006), 446-469.

[9] Kyoto Protocol to the United Nations Framework Convention on Climate Change, 1997. United Nations (UN), New York, NY, USA.

[10] Calm J. M., The next generation of refrigerants - Historical review, considerations, and outlook, Int. J. Ref. 31 (2008) 1123-1133.

[11] Horrocks, P.,EU F-gases Regulation and MAC Directive, ECCP-1 Review. European Commission Environment Directorate, Brussels, Belgium, (2006).

[12] Regulation (EC) No 842/2006 of the European Parliament and of the Council of 17 May 2006 on certain fluorinated greenhouse gases, Official J. of E.U., 49, (2006), L161.

[13] "Electrical Energy Reduction in Refrigeration and air Conditioning", Ken Landymore, Report Smartcool System Inc., 2007.

[14] J. M.Calm, 2002, Emissions and environmental impacts from air-conditioning and refrigeration systems, International Journal of Refrigeration, vol. 25, pp. 293-305.

[15] "Annual Energy Review 2010" Report DOE/EIA-0384 (2010), October 2011. US Department of Energy,USA.

[16] J. M.Calm, D.A. Didion, 1998, Trade-off in refrigerant selections: past, present, and future, International Journal of Refrigeration, vol. 21, pp. 308-321.

[17] J. M.Calm, 2006, Comparative efficiencies and implications for greenhouse gas emissions of chiller refrigerants, International Journal of Refrigeration, vol. 29, pp. 833-841.

[18] J.R. Sand, S.K. Fisher, V.D. Baxter, 1997, Energy and global warming impacts of HFC refrigerants and emerging technologies, Report sponsored by alternative fluorocarbons environmental acceptability study (AFEAS), U.S.Department of Energy, www.afeas.org.

[19] J.R. Sand, Fisher S.K., Baxter V.D., 1999, TEWI analysis: its utility, its shortcomings, and its results. Proceedings of the Taipei international conference on atmospheric protection.

[20] Third assessment report, Integovernative Panel on Climate Change, 2001, http://www.ipcc.ch/.

[21] Guideline method of calculating TEWI, 2006, British Refrigeration Association, Table 3.

[22] R. Maykot, G.C. Weber, R.A. Maciel, 2004, Using the TEWI methodology to evaluate alternative refrigerants technologies, International Refrigeration and Air Conditioning Conference at Purdue, July $12-15$.

[23] S. Malla, 2009, $\mathrm{CO}_{2}$ emissions from electricity generation in seven Asia-Pacific and North America countries: a decomposition analysis, Energy Policy, vol. 35, pp.5938-5952.

[24] R. Quadrelli, S. Peterson, 2007, The energy-climate challenge: recent trends in $\mathrm{CO}_{2}$ emissions from fuel combustion, Energy Policy, vol. 35, pp. 5938-5952.

[25] C. Weber, Koyama M., S. Kraines, 2006, $\mathrm{CO}_{2}$ emissions reductions potential and costs of decentralized energy system for providing electricity, cooling and heating in an officebuilding in Tokyo, Energy vol. 31, pp. 3041-3061. 
[26] C.S. Psomopoulos, I. Skoula, C. Karras, A. Chatzimpiros, M. Chionidis, 2010, Electricitysavings and $\mathrm{CO}_{2}$ emissions reduction in buildings sector: how important the network losses are I the calculation?, Energy, vol. 35, pp. 485-490.

[27] Jabaraj D.B., Narendran A., Mohan Lal D., Renganarayanan S., Evolving an optimal composition of HFC407C/HC290/HC600a mixture as an alternative to HCFC22 in window air conditioners, Int. J. of Th. Sci. 46 (2007) 276-283.

[28] Sarkar J., Cycle parameter optimization of vortex tube expansion transcritical CO2 system, Int. J. of Th. Sci. 48 (2009) 1823-1828.

[29] C. Aprea, A. Greco, 1998, An experimental evaluation of the greenhouse effect in R22 substitution, Energy Conversion \& Management, vol.39, n.9, pp.877-887.

[30] Greco A., Mastrullo R., Palombo A., 1997, R407C as an alternative to R22 in vapour compression plant: an experimental study, Int. Journ. of Energy Resaerch, vol. 21, pp.1087-1098.

[31]DuPont, Retrofit guidelines for DuPontTM Isceon ${ }^{\circledR}$ M029 (R422D) Refrigerant, Techincal Information art.46, http://www.refrigerants.dupont.com (2007)

[32]Arora A., Sachdev H.L., Thermodynamics analysis of R422 series refrigerants as alternative refrigerants to HCFC22 in a vapor compression refrigeration system; 33 (8), (2009) 753-65.

[33]Lazzarin R., Noro M., Experimental comparison of electronic and thermostatic expansion valves performances in an air conditioning plant, Int. J. Ref., 31,(2008),113-118.

[34]Jabaraj D.B., Narendran A., Mohan Lal D., Renganarayanan S., Evolving an optimal composition of HFC407C/HC290/HC600a mixture as an alternative to HCFC22 in window air conditioners, Int. J. of Th. Sci. 2007; 46:276-283

[35] C. Aprea, A. Maiorino, 2011, An experimental investigation of the global environmental impact of the R22 retrofit with R422D, Energy, vol. 36, pp.1161-1170.

[36] Commission Directive 94/2/EC of 21 January 1994 implementing Council Directive 92/75/EEC with regard to energy labeling of household electric refrigerators, freezers and their combinations.

[37] R.J. Moffat,1985, Using uncertainty analysis in the planning of an experiment, Trans. ASME, J. Fluids Eng., Vol. 107, pp. 173-178.

[38] C. Aprea, A. Maiorino, 2009, Transcritical CO2 refrigerator and sub-critical R134a refrigerator: a comparison of the experimental results, Int. Jour. of Energy Research, vol.33, pp.1040-1047.

[39] P. Nekså, 2002, $\mathrm{CO}_{2}$ heat pump systems, Int. J. Refrigeration, vol. 25, pp.421-427.

[40] C. Aprea, A. Maiorino, 2008, An experimental evaluation of the transcritical $\mathrm{CO}_{2}$ refrigerator performances using an internal heat exchanger, Int. Journal of Refrigeration, vol. 31, pp. 1006-1011.

[41] C. Aprea, A. Maiorino, 2009, Heat rejection pressure optimization for a carbon dioxide split system: an experimental study, Applied Energy, vol. 861, pp. 2373-2380.

[44] A. Fartaj, D.S.K. Ting, W.Yang, 2004, Second law analysis of the transcritical $\mathrm{CO}_{2}$ refrigeration cycle, Energy Conversion \& management, Vol.45, pp.2269-2281.

[45] Y.B. Tao, Y..L. He, W.Q. Tao, 2010, Exergetic analysis of a transcritical residential airconditioning system based on experimental data, Applied Energy, vo. 87, pp.3065-3072.

[46] Regulation (EC) 1999/100/CE. 